\title{
Interleukin-6 inhibition for treatment of rheumatoid arthritis: A review of tocilizumab therapy
}

\author{
Aarat M Patel ${ }^{1,2}$ \\ Larry W Moreland' \\ 'Division of Rheumatology and \\ Clinical Immunology, University \\ of Pittsburgh, Pittsburgh, PA, USA; \\ ${ }^{2}$ Division of Pediatric Rheumatology, \\ Children's Hospital of Pittsburgh, \\ Pittsburgh, PA, USA
}

This article was published in the following Dove Press journal:

Drug Design, Development and Therapy

7 October 2010

Number of times this article has been viewed

\begin{abstract}
The dawn of the biologic era has been an exciting period for clinical research and patient care in rheumatoid arthritis (RA). Targeted biologic therapies have changed the outcome of this disease and made remission a realistic outcome for many patients. Tocilizumab (TCZ, Actemra $^{\circledR}$ ), is a humanized monoclonal antibody against the interleukin 6 receptor and has been approved in many countries for the treatment of moderate to severe RA. There have been a number of important clinical trials demonstrating the efficacy of TCZ in active rheumatoid arthritis. This review summarizes the data on efficacy, patient-reported outcomes, adverse events, and safety from some of these trials. Current trends in clinical practice will be discussed. It is difficult to place TCZ and many new medications in the algorithm of treatment at present. However, the next few years will hopefully reveal their role as we better define abnormal immune processes in individuals with RA.
\end{abstract}

Keywords: tocilizumab, rheumatoid arthritis, interleukin-6, biologics

\section{Introduction}

Rheumatoid arthritis (RA) is a chronic systemic disease, with complex genetic and environmental factors acting together to cause inflammation and damage. This primarily occurs in the synovium and joint tissues, with typical clinical features being swollen painful joints and stiffness. Important extra-articular features include lung and cardiovascular disease. There is other organ involvement, and manifestations include anemia, fatigue, skin nodules, neuropathy, ocular disease, splenomegaly, vasculitis, and pleuropericarditis. ${ }^{1,2}$ There is a prevalence range of $0.5 \%-1.0 \%$ in many populations, with women being affected up to three times as often as men. The annual incidence of RA has been shown to be about 30 per 100,000. Peak onset of the disease is at $30-55$ years, but it can occur at any age. ${ }^{2,3}$

To prevent joint damage, disability, economic loss, and mortality, clinicians prefer aggressive therapy, with a common goal of remission. Despite the widespread use of disease-modifying antirheumatic drugs (DMARDs) and biologic agents in various combinations, some patients still have persistently active disease. Although there are various guidelines for treatment, ${ }^{4-6}$ each patient is managed individually, based on disease activity, prognostic signs, and functional impairment. There is a strong recommendation to use a biologic agent only after failure of nonbiologic DMARDs, unless high disease activity is present with poor prognostic signs. ${ }^{5,6}$ There are no consensus guidelines on which biologic agents to use after failure of a DMARD. However, there are trends in practice that are influenced by previous experience, tolerability, drug availability, and cost or insurance issues.
Correspondence: Aarat M Patel University of Pittsburgh, Children's Hospital, Suite 3000, 400 45th Street, Pittsburgh, PA I520I, USA

$\mathrm{Tel}+|\mathrm{I}| 2692508 \mid$

Fax +I 4126925054

Email aarat.patel@chp.edu 
Interleukin (IL)-6 is a pleiotropic proinflammatory cytokine that is produced by multiple cell types, but of importance in RA, it induces osteoclast differentiation and can be responsible for joint destruction. Increased serum and synovial fluid levels of IL-6 are correlated with RA disease activity. ${ }^{7,8}$

\section{DMARDS available}

Methotrexate (MTX) is the dominant small-molecule or nonbiologic DMARD, ${ }^{9}$ and is usually used as monotherapy or in combination with other DMARDs as a first-line agent in RA. There are guidelines in the $\mathrm{UK}^{4}$ that support combination DMARDs (MTX and at least one other DMARD) as firstline therapy; however, others feel that there is no significant advantage to this approach. ${ }^{10,11}$ DMARDS that are used alone or as combination therapy with or without MTX include leflunomide, sulfasalazine, and hydroxychloroquine. Gold agents, azathioprine, and cyclosporine are rarely used anymore. Nonsteroidal anti-inflammatory medications, as well as corticosteroids, are used in conjunction with DMARDs. Low-dose long-term corticosteroids are effective in some patients. ${ }^{12,13}$ Individuals with persistently active disease after a single DMARD or a combination of DMARDs are given a biologic agent. Some individuals with moderate to severe disease are given a combination of DMARD (such as MTX) plus a biologic agent as first-line therapy. MTX as monotherapy or in combination with small-molecule DMARDs has been shown to be effective as initial treatment, demonstrating a good outcome without the need for biologic therapy in some patients. ${ }^{14-16}$ The cost-effectiveness of biologics as first-line therapy is not as high as for conventional DMARDs as first-line therapy. ${ }^{17}$

In addition to the DMARDs discussed above, there are nine biologic therapies approved for RA in the US ${ }^{18}$ and Europe. ${ }^{19}$ Etanercept is an IgG fusion protein produced through the recombinant soluble p75 receptor for tumor necrosis factor-alpha (TNF- $\alpha$ ). Infliximab is a mouse-human monoclonal antibody against TNF- $\alpha$. Adalimumab is a fully human TNF- $\alpha$ monoclonal antibody. Many trials have shown the effectiveness of these three biologics that were the first to be approved for RA. ${ }^{14,15,20-26}$ When one of these agents fails or has to be discontinued, the choice of second-line biologic is up to the clinician, because it has been seen that efficacy varies on an individual basis. ${ }^{27}$ Data show that there is a high rate of continuation of the second agent when switching from one TNF- $\alpha$ inhibitor to another. ${ }^{28,29}$

There are newer therapies available that are used after the initial biologic fails due to persistently active disease, or at times these may even be used as the initial biologic. Certolizumab pegol, a PEGylated Fab' fragment on a humanized monoclonal TNF- $\alpha$ inhibitor antibody, was recently approved and is effective in moderate to severe RA. ${ }^{30-32}$ Golimumab, a fully human monoclonal antibody against TNF- $\alpha$, can be administered monthly. ${ }^{33}$ Abatacept, a fusion protein (cytotoxic $\mathrm{T}$ lymphocyte-associated antigen 4 fused to immunoglobulin) inhibits the costimulation of $\mathrm{T}$ cells successfully in patients refractory to DMARDs and other biologics. ${ }^{34-36}$ Rituximab, a monoclonal antibody to CD20, depletes B lymphocytes and is used in a variety of inflammatory disorders, including RA $\cdot{ }^{37}$ Anakinra is an IL-1 antagonist approved for RA and is given as a daily injection, so is less convenient and has a higher risk of injection site reactions than the TNF- $\alpha$ inhibitors. ${ }^{38,39}$

Despite the currently available therapies, there are patients who do not respond to DMARDs and biologics, or encounter problems with adverse effects and tolerance. For this reason, there is a continued demand for new therapies. Some that are currently in development and at the trial stages are agents targeting IL-1, cytokines from the TNF superfamily, IL-15, IL-17, B lymphocytes, IL-12/IL-23, SyK kinase, JAK3 kinase, and inhibitors of osteoclastogenesis. ${ }^{40-51}$

\section{Chemistry and mechanism of action}

Tocilizumab (TCZ) has a molecular weight of about $148 \mathrm{kDa}$ and is a recombinant humanized antihuman IL-6 receptor monoclonal antibody of the IgG1 $\kappa$ subclass with a typical $\mathrm{H}_{2} \mathrm{~L}_{2}$ polypeptide structure. The four polypeptide chains are linked intra- and intermolecularly by disulfide bonds; heavy chains consist of 448 amino acids and light chains consist of 214 amino acids. ${ }^{52}$

IL-6 is a pleiotropic proinflammatory cytokine produced by synovial and endothelial cells, so is produced in areas affected by inflammation. It is produced by T cells, B cells, lymphocytes, monocytes, and fibroblasts, and has a role in $\mathrm{T}$ cell activation and immunoglobulin secretion. IL-6 is involved in hepatic acute-phase protein synthesis and stimulation of hematopoietic precursor cell proliferation and differentiation. IL-6 signal transduction is mediated by membrane-bound and soluble IL-6 receptors. TCZ binds to both of these receptors and inhibits signaling via this route..$^{52,53}$

\section{Pharmacodynamics}

Studies of TCZ $4 \mathrm{mg} / \mathrm{kg}$ and $8 \mathrm{mg} / \mathrm{kg}$ have shown increased rheumatoid factor, erythrocyte sedimentation rate, C-reactive protein, and serum amyloid $\mathrm{A}$, as well as increased 
hemoglobin. Greater changes were observed with $8 \mathrm{mg} / \mathrm{kg}$. Healthy individuals given TCZ (2-28 mg/ $\mathrm{kg})$ showed a decrease in absolute neutrophil counts (nadir 3-5 days). RA patients followed a similar pattern. ${ }^{52}$

\section{Pharmacokinetics and metabolism}

A population analysis of 1793 RA patients treated with TCZ ( $4 \mathrm{mg} / \mathrm{kg}$ and $8 \mathrm{mg} / \mathrm{kg}$ every four weeks for 24 weeks) was used to determine pharmacokinetics, which are similar between healthy individuals and RA patients. Age, gender, and race did not affect the pharmacokinetics of TCZ. ${ }^{52}$

Clearance was $0.29 \pm 0.10 \mathrm{~mL} / \mathrm{h} / \mathrm{kg}$ with the mean apparent terminal half-life being $152 \pm 59$ hours after a single $10 \mathrm{mg} / \mathrm{kg}$ dose. Clearance decreased with increasing doses. Pharmacokinetic parameters did not change with time. Maximum concentration increased in a dose-proportional manner. There was then a more dose-proportional (4 mg/kg and $8 \mathrm{mg} / \mathrm{kg}$ every four weeks) increase seen in the area under the curve (AUC) and trough concentration. Comparing the $8 \mathrm{mg} / \mathrm{kg}$ with the $4 \mathrm{mg} / \mathrm{kg}$ dose showed an AUC 2.7-fold higher and trough levels 6.5 -fold higher than predicted. ${ }^{52}$

The TCZ $4 \mathrm{mg} / \mathrm{kg}$ dose every four weeks produced a predicted mean \pm standard deviation (SD) steady-state AUC, trough concentration, and peak level of $13,000 \pm 5800 \mu \mathrm{g} \cdot \mathrm{h} / \mathrm{mL}$, $1.49 \pm 2.13 \mu \mathrm{g} / \mathrm{mL}$ and $88.3 \pm 41.4 \mu \mathrm{g} / \mathrm{mL}$, respectively. The accumulation ratios for AUC, peak levels, and trough levels were $1.11,1.02$, and 1.96, respectively. All three parameters were higher with increased body weight. TCZ doses higher than $800 \mathrm{mg}$ per infusion are not recommended. The TCZ $8 \mathrm{mg} / \mathrm{kg}$ dose every four weeks had a predicted mean steady-state $\mathrm{AUC}$, and peak and trough levels of $35,000 \pm 15,500 \mu \mathrm{g} \cdot \mathrm{h} / \mathrm{mL}, 9.74 \pm 10.5 \mu \mathrm{g} / \mathrm{mL}$, and $183 \pm 85.6 \mu \mathrm{g} / \mathrm{mL}$, respectively. The accumulation ratios for AUC, and peak levels and trough levels were 1.22, 1.06, and 2.35, respectively. All three parameters were higher with increased body weight. ${ }^{52}$

Study data show that RA patients had a central volume of distribution of $3.5 \mathrm{~L}$ and a peripheral volume of distribution of $2.9 \mathrm{~L}$, resulting in a volume of distribution at steady state of 6.4 L. The total clearance of TCZ is concentration-dependent, and is determined by linear and nonlinear clearance. Linear clearance is estimated to be $12.5 \mathrm{~mL} / \mathrm{h}$ from population analysis. Low TCZ doses utilize nonlinear clearance and, because this becomes saturated at higher doses, linear clearance drives overall elimination. At steady state, the elimination half-life can be from 11 days $(4 \mathrm{mg} / \mathrm{kg}$ every four weeks) to 13 days ( $8 \mathrm{mg} / \mathrm{kg}$ every four weeks, and is therefore concentration-dependent. ${ }^{52}$
There are no formal studies of the effect of hepatic or renal impairment on the pharmacokinetics of TCZ. Patients given TCZ had normal renal function, and there does not appear to be a need to adjust the dose for mild renal impairment. ${ }^{52}$

IL-6 reduces mRNA expression for several cytochrome (CYP)450 isoenzymes (CYP1A2, CYP2B6, CYP2C9, CYP2C19, CYP2D6, and CYP3A4) in vitro, and this is reversed by coincubation with TCZ at clinically relevant concentrations. IL-6 signal inhibition may restore CYP450 to higher levels, thus increasing metabolism of drugs that are CYP450 substrates. Monitoring is recommended when a patient is taking a medication with a narrow therapeutic index (CYP450 substrate). Simvastatin and omeprazole are examples of medications for which TCZ administration may alter exposure. $^{52}$

\section{Clinical efficacy}

There are six fully published Phase III studies ${ }^{54-59}$ supporting use of TCZ in the treatment of RA. There are also other studies, including important Phase I, ${ }^{60} \mathrm{II}^{61-63}$ and III ${ }^{64}$ evaluations of response to TCZ by patients with active RA. These studies are reviewed briefly, followed by a more indepth summary of the fully published Phase III studies (Tables 1-6) for TCZ as monotherapy and in combination therapy.

\section{Phase I, II, and III studies}

A Phase I study ${ }^{60}$ explored the safety and efficacy of TCZ in RA patients. This was a randomized, double-blind, placebocontrolled, dose-escalation trial in 45 patients with active RA. The single TCZ dose given to the study group was $0.1,1,5$, or $10 \mathrm{mg} / \mathrm{kg}$. At week 2, a significant treatment difference was seen with the $5 \mathrm{mg} / \mathrm{kg}$ dose in regard to the American College of Rheumatology 20\% improvement criteria (ACR20) ${ }^{65}$ and disease activity score (DAS28) ${ }^{66}$ response. Diarrhea was the most common adverse event, with most adverse events being equal in both groups. This was the first randomized trial to show that IL-6 inhibition significantly improved signs, symptoms, and acute-phase reactions in RA patients. ${ }^{60}$

A Phase I/II study ${ }^{61}$ was conducted to evaluate the safety and efficacy ofTCZ in refractory RA. This was a multicenter, double-blind, placebo-controlled trial in 164 patients in whom the study doses were $4 \mathrm{mg} / \mathrm{kg}$ and $8 \mathrm{mg} / \mathrm{kg}$ administered for three months. A dose-dependent reduction in ACR20 response for the $8 \mathrm{mg} / \mathrm{kg}$ dose $(78 \%, P<0.001)$ was higher than for the $4 \mathrm{mg} / \mathrm{kg}$ dose $(57 \%)$ or placebo $(11 \%)$ at three months. A superior response was seen in the $8 \mathrm{mg} / \mathrm{kg}$ group for ACR50/ACR70 compared with placebo. 
Table I Baseline characteristics from published Phase III trials on tocilizumab monotherapy in rheumatoid arthritis

\begin{tabular}{|c|c|c|c|c|c|c|}
\hline & \multicolumn{2}{|c|}{ SAMURAI ${ }^{54}$ (52 wks) } & \multicolumn{2}{|c|}{ SATORI55 (24 wks) } & \multicolumn{2}{|c|}{ AMBITION 56 (24 wks) } \\
\hline \multirow[t]{2}{*}{ Criteria } & $\begin{array}{l}\text { Inclusion: } \\
5 \mathrm{yrs}, \mathrm{SJC} \\
30 \mathrm{~mm} / \mathrm{h} \text { ar } \\
\text { Inadequate } \\
\text { immunosup } \\
\text { Lymphocyt } \\
\text { Platelets } \geq \\
\text { Exclusion } \\
\text { and/or leflu } \\
\text { Ist dose, C } \\
\text { immunosup } \\
\text { exchange a } \\
\text { Ist dose, S } \\
\text { Significant } \\
\text { infection re } \\
\text { prior to Is }\end{array}$ & $\begin{array}{l}\text { RA } \geq 6 \text { mos - } \\
C \geq 6, \text { ESR } \geq \\
20 \mathrm{mg} / \mathrm{l}, \\
0 \geq \mathrm{I} \text { DMARD or } \\
\text { WBC } \geq 3.5 \times 10^{9} / /, \\
0.5 \times 10^{9}, \\
\text { anti-TNF } \\
3 \text { mos prior to } \\
\text { ose and type of } \\
\text { DMARDs, plasma } \\
\leq 4 \text { wks prior to } \\
\text { gic reaction, } \\
\text { t disease, Active } \\
\text { dication } \leq 4 \text { wks }\end{array}$ & $\begin{array}{l}\text { Exclusion } \\
<0.5 \times 10^{5} \\
\text { Platelets } \\
\text { agent or le } \\
\text { prior to st } \\
\text { or ALT or } \\
\text { ULN, Acti } \\
\text { or HBs ant } \\
\text { Significant } \\
\text { respiratory } \\
\text { renal, hepa }\end{array}$ & $\begin{array}{l}\text { cyte count } \\
3.5 \times 10^{9} / \mathrm{l}, \\
\text { led anti-TNF } \\
\text { e } \leq 12 \text { wks } \\
\text { s IV RA, AST } \\
r>1.5 \text {-fold } \\
\text { on, HCV ab } \\
\text { eaction to MTX, } \\
\text { cardiac, blood, } \\
\text { gic, endocrine, } \\
\text { ), Alcoholic }\end{array}$ & $\begin{array}{l}\text { Inclusion: } \\
\text { to severe } \\
\text { disease (SJ } \\
\text { TJC } \geq 8 \text { an } \\
\text { or CRP } \geq \\
\text { of NSAID } \\
\text { glucocortic } \\
\text { Exclusion } \\
\text { concurrent } \\
\text { Failed anti- } \\
\text { use } \leq 6 \text { mo } \\
\text { MTX adver } \\
\text { efficacy in }\end{array}$ & $\begin{array}{l}\text { Moderate } \\
\text { os, Active } \\
28 \mathrm{~mm} / \mathrm{h} \\
\text { Stable dose } \\
\text { wks } \\
\text { ctive TB, } \\
\text { t, MTX } \\
\text { to study, } \\
\text { or lack of }\end{array}$ \\
\hline & $\begin{array}{l}\text { DMARD }^{a} \\
(n=143)\end{array}$ & $\begin{array}{l}\text { TCZ } 8^{b} \\
(n=157)\end{array}$ & $\begin{array}{l}\text { MTXc } \\
(n=64)\end{array}$ & $\begin{array}{l}\text { TCZ } 8^{b} \\
(n=6 I)\end{array}$ & $\begin{array}{l}M^{\prime} X^{d} \\
(n=284)\end{array}$ & $\begin{array}{l}\text { TCZ } 8^{e} \\
(n=286)\end{array}$ \\
\hline Age, years & 53.1 & 52.9 & 50.8 & 52.6 & 50.0 & 50.7 \\
\hline Female, \% & 82 & 80 & 75 & 90 & 79 & 83 \\
\hline RA, years & 2.4 & 2.2 & 8.7 & 8.5 & 6.2 & 6.4 \\
\hline DAS28 (2-10) & 6.4 & 6.5 & 6.2 & 6.1 & 6.8 & 6.8 \\
\hline CRP mg/l & 49 & 47 & 32 & 30 & 31 & 30 \\
\hline $\mathrm{ESR} \mathrm{mm} / \mathrm{h}$ & 71.0 & 70.8 & 51.9 & 51.9 & 49.4 & 49.9 \\
\hline TJC & $14.4 / 49$ & $15.3 / 49$ & $14.2 / 49$ & $13.8 / 49$ & $31.1 / 68$ & $31.8 / 68$ \\
\hline SJC & $11.9 / 46$ & $12.5 / 46$ & $12.7 / 46$ & $12.4 / 46$ & $19.2 / 66$ & $19.1 / 66$ \\
\hline HAQ-DI (0-3) & - & - & - & - & 1.5 & 1.6 \\
\hline Pain VAS & - & - & - & - & 61.5 & 58.7 \\
\hline Patient VAS & - & - & - & - & 65.6 & 63.9 \\
\hline Phys. VAS & - & - & - & - & 63.6 & 63.0 \\
\hline TSS & 30.6 & 28.3 & - & - & - & - \\
\hline Erosion score & 13.9 & 13.8 & - & - & - & - \\
\hline JSN score & 16.7 & 14.5 & - & - & - & - \\
\hline VEGF pg/ml & - & - & 730.8 & 711.3 & - & - \\
\hline DMARD/TNF use & $2.8(I-7)^{f}$ & $2.7(\mathrm{I}-7)^{f}$ & $3.6(I-8)^{f}$ & $3.3(I-8)^{f}$ & $\mathrm{I} . \mathrm{I} \pm \mathrm{I} .4^{\mathrm{g}}$ & $1.2 \pm 1.3^{g}$ \\
\hline
\end{tabular}

Notes: Except where indicated otherwise, values are the mean. A blank represents no reported value. ${ }^{\mathrm{a} D M A R D}$ group: MTX (30\%), MTX+ DMARD (37\%) or DMARD and/ or immunosuppressant's other than MTX (22\%) + TCZ Placebo; ' ${ }^{\mathrm{T}} \mathrm{TCZ} 8 \mathrm{mg} / \mathrm{kg}+$ MTX Placebo; 'MTX + TCZ Placebo; ${ }^{\mathrm{d}} \mathrm{MTX}$ (7.5-20 mg escalating dose); ${ }^{\mathrm{e}} \mathrm{TCZ} 8 \mathrm{mg} / \mathrm{kg}$; 'Number (range) of failed DMARDS; gMean \pm SD of DMARD and anti-TNF's used previously.

Abbreviations: RA, rheumatoid arthritis; DMARD, disease-modifying antirheumatic drug; TNF, tumor necrosis factor; DAS28, disease activity score 28; SJC, swollen joint count; TJC, tender joint count; ESR, erythrocyte sedimentation rate; CRP, C-reactive protein; WBC, white blood cell; TCZ, tocilizumab; MTX, methotrexate; TSS, total sharp score; NSAID, nonsteroidal anti-inflammatory drug; AST, aspartate aminotransferase; ALT, alanine aminotransferase; Cr, Creatinine; ULN, upper limit of normal; LLN, lower limit of normal; TB, tuberculosis; HCV, hepatitis C virus; HBs, hepatitis B surface; GI, gastrointestinal; JSN, joint space narrowing; VEGF, vascular endothelial growth factor; VAS, visual analog scale (0-100 mm); HAQ-DI, Health Assessment Questionnaire Disability Index.

Thirty-one patients withdrew from this study, including 25 from the placebo group. Important adverse events comprised a cholesterol increase in $44 \%$ of patients, liver function disorders, and decreased white blood cell counts. ${ }^{61}$

CHARISMA $^{62}$ was a randomized, 16-week, Phase II, multicenter double-blind, placebo-controlled European trial in 359 patients which was done to establish the safety and efficacy of repeat infusions of TCZ in RA not fully responsive to MTX. There were seven treatment arms comprising TCZ $2 \mathrm{mg} / \mathrm{kg}$, $4 \mathrm{mg} / \mathrm{kg}$, or $8 \mathrm{mg} / \mathrm{kg}$, either as monotherapy or combination therapy with MTX, and MTX plus placebo. A statistically significant $(P<0.05)$ ACR20 was seen in $61 \%$ and $63 \%$ of those on monotherapy (TCZ $4 \mathrm{mg} / \mathrm{kg}$ and $8 \mathrm{mg} / \mathrm{kg}$, respectively) and in $63 \%$ and $74 \%$ of those with the same TCZ dose plus MTX, respectively, compared with $41 \%$ receiving MTX plus placebo. As early as week 4, all study drug groups achieved a dose-related reduction in DAS28, except for the group receiving TCZ $2 \mathrm{mg} / \mathrm{kg}$ monotherapy. The inclusion criteria required patients to have been on MTX for at least four weeks; a high proportion of patients 
Table 2 Baseline characteristics from published Phase III trials on tocilizumab combination therapy in rheumatoid arthritis

\begin{tabular}{|c|c|c|c|c|c|c|c|c|}
\hline & \multicolumn{3}{|c|}{ OPTION $^{57}$ (24 wks) } & \multicolumn{3}{|c|}{ RADIATE $^{58}$ (24 wks) } & \multicolumn{2}{|c|}{ TOWARD 59 (24 wks) } \\
\hline \multirow[t]{3}{*}{ Criteria } & \multicolumn{3}{|c|}{$\begin{array}{l}\text { Inclusion: Adult, RA } \geq 6 \mathrm{mos}, \text { Active disease } \\
(\mathrm{SJC} \geq 6+\mathrm{TJC} \geq 8 \text { or ESR } \geq 28 \mathrm{~mm} / \mathrm{hr} \text { or } \\
\mathrm{CRP}>10 \mathrm{mg} / \mathrm{l}) \text {, Received } \mathrm{MTX} \geq 12 \mathrm{wks} \\
\text { (stable dose } 10-25 \mathrm{mg} / \mathrm{wk} \geq 8 \mathrm{wks} \text { ) with } \\
\text { inadequate response prior to study, } \\
\text { Discontinued all other DMARDs (etanercept } \\
\geq 2 \text { wks, infliximab or adalimumab } \geq 8 \mathrm{wks} \text {, } \\
\text { leflunomide } \geq 12 \text { wks or } \geq 4 \text { wks with } \\
\text { colestyramine washout, anakinra } \geq \mathrm{lwk} \text { ), } \\
\text { Stable dosage of NSAIDs and/or oral } \\
\text { steroids } \geq 6 \text { wks }\end{array}$} & \multicolumn{3}{|c|}{$\begin{array}{l}\text { Inclusion: Age } \geq 18, \text { Moderate to severe } \\
\text { RA } \geq 6 \mathrm{mos}(\mathrm{SJC} \geq 6+\mathrm{TJC} \geq 8 \text { ), } \\
\text { ESR } \geq 28 \mathrm{~mm} / \mathrm{h} \text { and } C R P \geq I \mathrm{mg} / \mathrm{dl} \text {, Failed } \\
\text { anti-TNF } \leq I \mathrm{yr}, \text { Discontinued etanercept } \\
\text { ( } \geq 2 \mathrm{wks} \text { ), infliximab or adalimumab ( } \geq 8 \mathrm{wks} \text { ), } \\
\text { leflunomide ( } \geq 12 \text { weeks) and all DMARDs } \\
\text { (except } M T X \text { ) prior to study }\end{array}$} & \multicolumn{2}{|c|}{$\begin{array}{l}\text { Inclusion: Age } \geq 18, \\
\text { Moderate to severe } \\
\text { RA } \geq 6 \mathrm{mos}, \mathrm{SJC} \geq 6+ \\
\text { TJC } \geq 8, \mathrm{ESR} \geq 28 \mathrm{~mm} / \mathrm{h} \\
\text { or CRP } \geq \mathrm{I} \mathrm{mg/dl,} \mathrm{Stable} \\
\text { dose of NSAID/oral } \\
\text { steroid } \geq 6 \text { wks, Stable } \\
\text { dose of DMARDs } \geq \\
8 \text { wks prior to study } \\
\text { (MTX, HCQ, SZA, LEF, } \\
\text { gold, AZA or combination } \\
\text { of } 2-3 \text { DMARDS) }\end{array}$} \\
\hline & \multicolumn{3}{|c|}{$\begin{array}{l}\text { Exclusion: Class IV RA, Other autoimmune } \\
\text { disease, significant systemic involvement of } \\
\text { RA, other inflammatory joint disease, } \\
\text { recurrent infections, AST or ALT > I.5 } \\
\text { ULN, Failed anti-TNF agent }\end{array}$} & \multicolumn{3}{|c|}{$\begin{array}{l}\text { Exclusion: Cell depleting agent treatment, } \\
\text { Uncontrolled medical conditions, Other } \\
\text { inflammatory disease, Class IV RA, Malignancy, } \\
\text { Recurrent infections, Active TB, Hepatitis B or } \\
\text { C, Immunodeficient, Hemoglobin }<8.5 \mathrm{~g} / \mathrm{dl} \text {, } \\
\text { leukopenia, neutropenia, thrombocytopenia, } \\
\text { Triglycerides }>10 \mathrm{mmol} / \mathrm{l} \text {, Liver disease }\end{array}$} & \multicolumn{2}{|c|}{$\begin{array}{l}\text { Exclusion: Failed anti- } \\
\text { TNF, cell depleting agent } \\
\text { treatment, Failed TB } \\
\text { screen per local practice }\end{array}$} \\
\hline & $\begin{array}{l}M^{\prime} X^{a} \\
(n=204)\end{array}$ & $\begin{array}{l}T C Z 4^{b} \\
(n=213)\end{array}$ & $\begin{array}{l}\text { TCZ } 8^{c} \\
(n=205)\end{array}$ & $\begin{array}{l}M^{\prime} X^{a} \\
(n=I 58)\end{array}$ & $\begin{array}{l}\operatorname{TCZ} 4^{\mathrm{b}} \\
(\mathrm{n}=161)\end{array}$ & $\begin{array}{l}\text { TCZ } 8^{c} \\
(n=\mid 70)\end{array}$ & $\begin{array}{l}\text { DMARD }^{d} \\
(n=4 \mid 3)\end{array}$ & $\begin{array}{l}\text { TCZ } 8^{e} \\
(n=803)\end{array}$ \\
\hline Age, years & 50.6 & 51.4 & 50.8 & 53.4 & 50.9 & 53.9 & 54 & 53 \\
\hline Female, \% & 78 & 82 & 85 & 79 & 81 & 84 & 84 & 81 \\
\hline RA, years & 7.8 & 7.4 & 7.5 & 11.4 & $\mathrm{II}$ & 12.6 & 9.8 & 9.8 \\
\hline DAS28 (2-10) & 6.8 & 6.8 & 6.8 & 6.80 & 6.78 & 6.79 & 6.6 & 6.7 \\
\hline CRP mg/l & 24 & 28 & 26 & 37 & 31 & 28 & 26 & 26 \\
\hline $\mathrm{ESR} \mathrm{mm} / \mathrm{h}$ & 49.7 & 49.2 & 51.2 & 54.6 & 51.3 & 49.1 & 49.2 & 48.2 \\
\hline TJC & $32.8 / 68$ & $33.2 / 68$ & $31.9 / 68$ & 30.4 & 31.3 & 31.7 & $29.1 / 66$ & $30.1 / 66$ \\
\hline SJC & $20.7 / 66$ & $20.0 / 66$ & $19.5 / 66$ & 18.9 & 19.5 & 18.9 & $18.7 / 68$ & $19.7 / 68$ \\
\hline HAQ-DI (0-3) & 1.5 & 1.6 & 1.6 & 1.7 & 1.7 & 1.7 & 1.5 & 1.5 \\
\hline SF-36 physical & 32.3 & 31.5 & 32.7 & - & - & - & - & - \\
\hline SF-36 mental & 39.1 & 40.1 & 40.9 & - & - & - & - & - \\
\hline FACIT (0-52) & 26.7 & 27 & 27.7 & - & - & - & - & - \\
\hline Pain VAS & 57.3 & 60.7 & 59.9 & 64.1 & 63.5 & 64.7 & 59 & 58 \\
\hline Patient VAS & 63.6 & 65.6 & 64.8 & 70.9 & 70.4 & 70.2 & 66 & 66 \\
\hline Phys. VAS & 63.7 & 63.6 & 64 & 67.5 & 66.5 & 66.4 & 63 & 64 \\
\hline MTX, mg & 14.8 & 14.7 & 14.5 & 16.5 & 16.2 & 15.7 & 15.0 & 14.7 \\
\hline DMARD use & $1.7 \pm 1.5^{f}$ & $1.5 \pm 1.4^{f}$ & $1.5 \pm 1.4^{f}$ & $2.1 \pm 1.6^{f}$ & $2.0 \pm 1.6^{f}$ & $1.9 \pm 1.7^{f}$ & $1.6 \pm 1.6^{8}$ & $1.6 \pm 1.6^{\mathrm{g}}$ \\
\hline TNF use & $19(9 \%)^{\mathrm{h}}$ & $21(10 \%)^{\mathrm{h}}$ & II $(55 \%)^{\mathrm{h}}$ & $\begin{array}{l}30.6 \% \\
39.4 \%^{\mathrm{i}} \\
29.4 \%^{\mathrm{k}}\end{array}$ & $\begin{array}{l}38.0 \%^{\mathrm{i}} \\
34.4 \%^{\mathrm{j}} \\
26.4 \%^{\mathrm{k}}\end{array}$ & $\begin{array}{l}38.3 \%^{i} \\
30.3 \%^{j} \\
31.4 \%^{k}\end{array}$ & & \\
\hline
\end{tabular}

Notes: Except where indicated otherwise, values are the mean. A blank represents no reported value. aMTX I0-25 mg + TCZ placebo; ${ }^{\mathrm{b}} \mathrm{TCZ} 4 \mathrm{mg} / \mathrm{kg}+\mathrm{MTX}$ I0-25 mg;

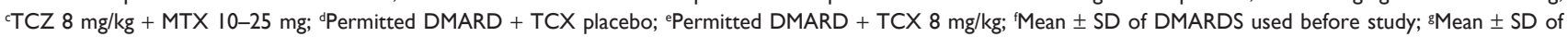
DMARD plus anti-TNF's used previously; 'Number (\%) of total that used anti-TNF's previously; 'Percent with previous Etanercept use; 'Percent with previous Adalimumab use; 'Percent with previous Infliximab use.

Abbreviations: RA, rheumatoid arthritis; SJC, swollen joint count; TJC, tender joint count; MTX, methotrexate; ESR, erythrocyte sedimentation rate; CRP, C-reactive protein; AST, aspartate aminotransferase; ALT, alanine aminotransferase; TNF, tumor necrosis factor; DMARD, disease-modifying antirheumatic drug; TB, tuberculosis; NSAID, nonsteroidal anti-inflammatory drug; ULN, upper limit of normal; HCQ, hydroxychloroquine; SZA, sulfasalazine; LEF, leflunomide; AZA, azathioprine; TCZ, Tocilizumab; DAS28, disease activity score 28; HAQ-DI, Health Assessment Questionnaire Disability Index; SF36, Short Form 36 (scored 0-I00); FACIT, Functional Assessment of Chronic Illness Therapy; VAS, visual analog scale (0-100 mm).

in the placebo group responded to MTX, demonstrating there was not an initial "incomplete response". Important adverse events were a rise in alanine transaminase, aspartate transaminase, total cholesterol, and triglyceride levels. There was a reduction in neutrophil levels. ${ }^{62}$

STREAM $^{63}$ was a long-term extension of the three-month study ${ }^{61}$ described above, and evaluated the safety and efficacy of TCZ $8 \mathrm{mg} / \mathrm{kg}$ monotherapy for five years in 143 patients. Forty-eight patients had withdrawn from the study (32 due to adverse events and one due to a poor response). The serious adverse event rate was 27.5 events per 100 patientyears, with 5.7 serious infections per 100 patient-years. ACR20, 50, and 70 responses at five years analyzed by last observation carried forward were $77 \%, 59 \%$, and $38 \%$, 
Table 3 Clinical response from Phase III trials on tocilizumab monotherapy in rheumatoid arthritis

\begin{tabular}{|c|c|c|c|c|c|c|}
\hline & \multicolumn{2}{|c|}{ SAMURAI $^{54}$ (52 wks) } & \multicolumn{2}{|c|}{ SATORI55 (24 wks) } & \multicolumn{2}{|c|}{ AMBITION $^{56}$ (24 wks) } \\
\hline & $\begin{array}{l}\text { DMARD }^{a} \\
(n=143)\end{array}$ & $\begin{array}{l}\text { TCZ } 8^{b} \\
(n=157)\end{array}$ & $\begin{array}{l}\text { MTXc } \\
(n=64)\end{array}$ & $\begin{array}{l}\text { TCZ 8 } \\
(n=61)\end{array}$ & $\begin{array}{l}\text { MTX } \\
(n=284)\end{array}$ & $\begin{array}{l}\text { TCZ 8e } \\
(n=286)\end{array}$ \\
\hline ACR20 & 34 & $78 *$ & 25 & $80 *$ & 53 & $70 *$ \\
\hline ACR50 & 13 & $64 *$ & 11 & $49 *$ & 34 & $44 * *$ \\
\hline ACR70 & 6 & $44 *$ & 6 & $30 *$ & 15 & $28^{*}$ \\
\hline DAS $28<2.6$ & 3 & $59 *$ & 1.6 & $43.1^{*}$ & 12 & $34^{\$}$ \\
\hline EULAR response & - & - & 39.7 & 96.6 & 64.8 & 82.2 \\
\hline CRP mg/l & - & - & - & - & -1.9 & $-2.8^{\$ \$}$ \\
\hline $\mathrm{ESR} \mathrm{mm} / \mathrm{h}$ & - & - & - & - & -16.1 & $-37.3^{\$ \$}$ \\
\hline TJC & - & - & - & - & -13.9 & $-17.2^{\$ \$}$ \\
\hline SJC & - & - & - & - & -8.2 & $-11.7^{\$ \$}$ \\
\hline HAQ-DI & - & - & - & - & -0.5 & $-0.7^{\$ \$}$ \\
\hline Pain VAS & - & - & - & - & -29.9 & -31.9 \\
\hline Pt. VAS & - & - & - & - & -30.7 & -34.5 \\
\hline Phys VAS & - & - & - & - & -31.7 & $-41.3^{\$ \$}$ \\
\hline TSS & 6.1 & $2.3^{\#}$ & - & - & - & - \\
\hline Erosion score & 3.2 & $0.9 *$ & - & - & - & - \\
\hline JSN score & 2.9 & $1.5^{\#}$ & - & - & - & - \\
\hline VEGF $\mathrm{pg} / \mathrm{ml}$ & - & - & -74.0 & $-346.9 *$ & - & - \\
\hline MHAQ $(\downarrow \geq 0.22)$ & 40 & $68^{*}$ & 34 & $67^{*}$ & - & - \\
\hline
\end{tabular}

Notes: ACR20, 50, 70, DAS28, EULAR response (good+moderate) and MHAQ are all shown as proportion of patients who achieved response CRP, ESR, TJC, SJC, HAQDI, VAS, TSS, erosion score, JSN score are all shown as mean change from baseline A blank represents no reported value. ${ }^{* P}<0.001$; ${ }^{* * P}=0.002 ;{ }^{*} P<0.01$; ${ }^{*} P<0.05$; ${ }^{\$} \mathrm{Odds}$ ratio vs MTX: 5.83 ; $95 \% \mathrm{Cl} 3.27$ to 10.40 ; $\$$ Superiority criteria met ${ }^{a}$ DMARD group: MTX (30\%), MTX + DMARD (37\%) or DMARD and/or immunosuppressant's other than MTX (22\%) + TCZ Placebo; ' ${ }^{\mathrm{T} C Z} 8 \mathrm{mg} / \mathrm{kg}+$ MTX Placebo; ${ }^{\mathrm{c} M T X}+\mathrm{TCZ}$ Placebo; $\mathrm{d}$ MTX (7.5-20 mg escalating dose); ${ }^{\mathrm{e}} \mathrm{TCZ} 8 \mathrm{mg} / \mathrm{kg}$.

Abbreviations: ACR20,50,70, American College of Rheumatology 20\%, 50\%, 70\% improvement criteria; DAS28, disease activity score 28 (<2.6 indicates remission); EULAR, European League Against Rheumatism (good+moderate); CRP, C-reactive protein; ESR, erythrocyte sedimentation rate; TJC, tender joint count; SJC, swollen joint count; HAQ-DI, Health Assessment Questionnaire-Disability Index (scored 0-3); TSS, Total Sharp score; VAS, visual analog scale (scored 0-I00 mm); JSN, joint space narrowing score; VEGF, vascular endothelial growth factor; MHAQ, Modified Health Assessment Questionnaire ( $\downarrow$ of $\geq 0.22$ is significant clinical improvement).

respectively. Corticosteroid doses were decreased in $89 \%$ of patients. A remission (disease activity score $<2.6$ ) was seen in $55 \%$ of patients. This was the first study to demonstrate the long-term safety and efficacy of TCZ monotherapy in DMARD-resistant disease. Total cholesterol elevation was seen throughout the study period, and there was one episode of ischemic heart disease in a patient who also had diabetes mellitus. Mean neutrophil counts decreased but remained in the normal range. There was no significant liver disease seen, despite slight elevations of mean alanine transaminase and aspartate transaminase values. ${ }^{63}$

LITHE $^{64}$ was a Phase III, international (15 countries), randomized, double-blind, placebo-controlled study of TCZ in patients with moderate to severe RA who remained on MTX despite inadequate response. Patients received TCZ $4 \mathrm{mg} / \mathrm{kg}$ or $8 \mathrm{mg} / \mathrm{kg}$, or placebo, with background MTX (52 weeks with rescue at 16 weeks if needed). All patients were to take TCZ $8 \mathrm{mg} / \mathrm{kg}$ for the second year unless they achieved $\geq 70 \%$ improvements in swollen joint count and tender joint count. A primary endpoint at week 104 was change from baseline in Genant-modified Total Sharp Score, which showed that there was significantly less radiographic progression in the TCZ $8 \mathrm{mg} / \mathrm{kg}$ group compared with placebo. There were significantly more TCZ $8 \mathrm{mg} / \mathrm{kg}$ patients than those on placebo with no radiographic progression $(P \leq 0.0001)$. The change from baseline Health Assessment Questionnaire-Disability Index (HAQ-DI ${ }^{67}$ was significant in both TCZ groups when compared with placebo. From the data presented, serious adverse events are comparable in all three groups, but the results of this study have not been fully published at the time of writing.

Fully published Phase III trials are now described. Baseline data, as well as inclusion and exclusion criteria, for each study are summarized in Tables 1 and 2. Some of the patients enrolled had a history of previous use and/or failure of DMARDs and TNF- $\alpha$ inhibitors, except for those in the AMBITION study. The duration of each trial ranges from 24-52 weeks. Important efficacy outcomes are summarized in Tables 3 and 4. ACR20 was the primary endpoint in all studies, except for SAMURAI, ie, the only study that investigated patients with early RA. All the studies described had the primary efficacy endpoint analyzed in the intentionto-treat population with last observation carried forward, except for AMBITION; noninferiority on the per-protocol 
Table 4 Clinical response from the Phase III trials on tocilizumab combination therapy in rheumatoid arthritis

\begin{tabular}{|c|c|c|c|c|c|c|c|c|}
\hline & \multicolumn{3}{|c|}{ OPTION $^{57}$ (24 wks) } & \multicolumn{3}{|c|}{ RADIATE $^{58}$ (24 wks) } & \multicolumn{2}{|c|}{ TOWARD $^{59}$ (24 wks) } \\
\hline & $\begin{array}{l}M^{M} X^{a} \\
(n=204)\end{array}$ & $\begin{array}{l}\text { TCZ 4 } \\
(n=213)\end{array}$ & $\begin{array}{l}\text { TCZ 8c } \\
(n=205)\end{array}$ & $\begin{array}{l}\text { MTX }^{a} \\
(n=158)\end{array}$ & $\begin{array}{l}\operatorname{TCZ} 4^{b} \\
(n=161)\end{array}$ & $\begin{array}{l}\text { TCZ 8c } \\
(n=170)\end{array}$ & $\begin{array}{l}\text { DMARD }^{d} \\
(n=4 \mid 3)\end{array}$ & $\begin{array}{l}\text { TCZ 8 } \\
(n=803)\end{array}$ \\
\hline ACR20 & 26 & $48 *$ & $59 *$ & 10.1 & $30.4^{* *}$ & $50.0 * *$ & 24.5 & $60.8^{*}$ \\
\hline ACR50 & 22 & $31 *$ & $44^{*}$ & 3.8 & $16.8 * *$ & $28.8 * *$ & 9.0 & $37.6 *$ \\
\hline ACR70 & 4 & $12^{*}$ & $22 *$ & 1.3 & 5.0 & $12.4^{\#}$ & 2.9 & $20.5^{*}$ \\
\hline DAS $28<2.6$ & 0.8 & $13 * *$ & $27^{*}$ & 1.6 & 7.6 & $30.1^{\#}$ & 3 & $30 *$ \\
\hline EULAR response & 33 & $62^{*}$ & $79 *$ & 16.5 & $46.5^{* *}$ & $67.7 * *$ & 38 & $80 *$ \\
\hline CRP mg/l & -3.5 & $-16.6^{* *}$ & $-25 . I^{*}$ & - & - & - & -2.7 & $-22^{*}$ \\
\hline $\mathrm{ESR} \mathrm{mm} / \mathrm{h}$ & -7.1 & $-25.5^{*}$ & $-39.5^{*}$ & - & - & - & -4.7 & $-35.6^{*}$ \\
\hline TJC & -7.4 & $-14.5^{*}$ & $-17.1 *$ & -0.3 & $-10.5 * *$ & $-14.8 * *$ & -8.5 & $-15.7^{*}$ \\
\hline SJC & -4.3 & $-8.5^{*}$ & $-10.5^{*}$ & -0.5 & $-6.8^{* *}$ & $-7.8 * *$ & -4.9 & $-10.3^{*}$ \\
\hline HAQ-DI & -0.34 & $-0.52^{\#}$ & $-0.055^{\#}$ & -0.05 & $-0.31 * *$ & $-0.39 * *$ & -0.2 & $-0.5^{*}$ \\
\hline SF-36 physical & 5.0 & $9.7 *$ & $9.5^{*}$ & - & - & - & 4.1 & $8.9 *$ \\
\hline SF-36 mental & 2.7 & $5.7^{\#}$ & $7.3^{\#}$ & - & - & - & 2.3 & $5.3^{*}$ \\
\hline FACIT & 4.0 & $7.3^{\#}$ & $8.6^{*}$ & - & - & - & 3.6 & $8.0^{*}$ \\
\hline Pain VAS & -14.0 & $-25.0 * *$ & $-29.8^{*}$ & - & - & - & - & - \\
\hline Pt. VAS & -17.8 & $-28.8^{* *}$ & $-32.7^{*}$ & - & - & - & - & - \\
\hline Phys VAS & -32.7 & $-38.3^{\# \#}$ & $-41.6 * *$ & - & - & - & - & - \\
\hline
\end{tabular}

Notes: ACR20, 50, 70, DAS28 and EULAR response (good+moderate) are all shown as proportion of patients who achieved response CRP, ESR, TJC, SJC, HAQDI, SF36, FACIT and VAS are all shown as mean change from baseline A blank represents no reported value. ${ }^{*} P<0.0001$; ${ }^{* * P}<0.001$; ${ }^{*} P<0.01$; ${ }^{\# * P}<0.05$; ${ }^{\mathrm{a}} \mathrm{MTX}$ I0-25 mg + TCZ

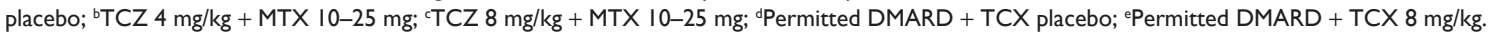

Abbreviations: ACR20,50,70, American College of Rheumatology 20\%, 50\%, 70\% improvement criteria; DAS28, disease activity score 28 ( $<2.6$ indicates remission); EULAR, European League Against Rheumatism (good+moderate); CRP, C-reactive protein; ESR, erythrocyte sedimentation rate; TJC, tender joint count; SJC, swollen joint count; HAQ, Health Assessment Questionnaire Disability Index (scored 0-3); SF36, Short Form 36 (scored 0-100); FACIT, Functional Assessment of Chronic Illness Therapy (scored 0-100); VAS, visual analog scale (scored 0-100 mm).

population and superiority on the intention to treat. The baseline characteristics and demographics in each study were not different between the study and placebo groups.

\section{Tocilizumab monotherapy in rheumatoid arthritis}

AMBITION $^{56}$ was a 24-week, Phase III, international (US, Canada, and Israel), randomized, double-blind, doubledummy, placebo-group study evaluating the efficacy and safety of TCZ monotherapy versus MTX in patients with active RA for whom previous therapy with MTX and/ or biologic agents had not failed. Patients were randomly assigned to two groups. One groups received TCZ $8 \mathrm{mg} / \mathrm{kg}$ intravenously every four weeks $(n=288)$ and the second group received MTX (escalating dose regimen, ie, initially $7.5 \mathrm{mg}$, increasing to $15 \mathrm{mg}$ at week 4 and $20 \mathrm{mg}$ at week 8) orally once weekly $(\mathrm{n}=288)$. There was a substudy arm which is not reviewed here. In total, 673 patients were randomized, with most completing the study (93\% in the TCZ group and $92 \%$ in the MTX group)

The primary endpoint was ACR20 response at week 24 . The primary efficacy analysis was a noninferiority comparison using the established per-protocol population (ACR20 $70.6 \%$ for TCZ versus $52.1 \%$ for MTX). Superiority of TCZ to MTX was shown in the intention-to-treat population as early as week 2, with an increase over time (ACR20 69.6\% for TCZ versus $52.5 \%$ for MTX, $P<0.001$ at week 24). Important secondary endpoints (Table 3 ) analyzed by superiority in the intention-to-treat population included the proportion of those with an ACR50/ACR70 response at week 24. Changes from baseline in DAS28, ${ }^{66}$ inflammatory markers, and HAQ-DI were assessed at week 24 . The proportion of those with clinical remission (DAS28 < 2.6) and a good/moderate European League Against Rheumatism (EULAR) response ${ }^{68}$ was also assessed. Pain and patient's global assessment of disease activity were measured using a visual analog scale. ${ }^{69}$

SATORI $^{55}$ was a 24 -week, multicenter, randomized, double-blind, placebo-controlled Japanese study evaluating the efficacy and safety of TCZ monotherapy in patients with active RA AND an inadequate response to MTX. Patients were randomly assigned to one of two groups. One group received TCZ $8 \mathrm{mg} / \mathrm{kg}$ intravenously every four weeks plus an MTX placebo weekly $(\mathrm{n}=61)$ and the second group received TCZ or placebo intravenously every four weeks plus MTX $8 \mathrm{mg}$ orally weekly $(\mathrm{n}=66)$. A total of 127 patients were randomized, with 88 completing the study ( $89 \%$ in the TCZ group and 52\% in the MTX group). 
Table 5 Adverse events from Phase III trials on tocilizumab monotherapy in rheumatoid arthritis

\begin{tabular}{|c|c|c|c|c|c|c|}
\hline & \multicolumn{2}{|c|}{ SAMURAI ${ }^{54}$ (52 wks) } & \multicolumn{2}{|c|}{ SATORI55 (24 wks) } & \multicolumn{2}{|c|}{ AMBITION $^{56}$ (24 wks) } \\
\hline & $\begin{array}{l}\text { DMARD }^{a} \\
(n=143)\end{array}$ & $\begin{array}{l}\text { TCZ } 8^{b} \\
(n=157)\end{array}$ & $\begin{array}{l}\text { MTXc }^{c} \\
(n=64)\end{array}$ & $\begin{array}{l}\text { TCZ } 8^{b} \\
(n=6 I)\end{array}$ & $\begin{array}{l}M^{\prime} X^{d} \\
(n=284)\end{array}$ & $\begin{array}{l}\text { TCZ } 8^{e} \\
(n=286)\end{array}$ \\
\hline Any adverse event & 82 & 89 & 72 & 92 & 78 & 80 \\
\hline Serious adverse event & 13 & 18 & 4.7 & 6.6 & 3 & 4 \\
\hline Related serious adverse event & - & - & - & - & I & I \\
\hline AE leading to discontinuation & - & - & - & - & 5 & 4 \\
\hline AE leading to dose modification & - & - & - & - & 22 & 19 \\
\hline \multirow[t]{2}{*}{ Death } & - & - & - & - & $<1$ & 1 \\
\hline & \multicolumn{2}{|c|}{ AE's in $\geq 5 \%$} & \multicolumn{2}{|c|}{ AE's in > $5 \%$} & \multicolumn{2}{|c|}{ AE's in $\geq 5 \%$} \\
\hline Infections and infestations & - & - & - & - & 37 & 34 \\
\hline Nasopharyngitis & 32 & 36 & 11 & 18 & 6 & 7 \\
\hline Upper respiratory tract infections & - & - & 6 & 5 & 5 & 7 \\
\hline Stomatitis & - & - & 0 & 12 & - & - \\
\hline Gastrointestinal disorders & - & - & - & - & 31 & 30 \\
\hline Nausea & I & 6 & - & - & 12 & 6 \\
\hline Vomiting & 3 & 5 & - & - & - & - \\
\hline Diarrhea & 9 & 8 & 2 & 7 & 5 & 5 \\
\hline MSK + CT disorders & - & - & - & - & 11 & 12 \\
\hline Skin and SC tissue disorders & - & - & - & - & 11 & 15 \\
\hline Rash & 4 & 11 & - & - & - & - \\
\hline Eczema & 4 & 6 & - & - & - & - \\
\hline Paronychia & $<1$ & 6 & - & - & - & - \\
\hline Nervous system disorders & - & - & - & - & 6 & 13 \\
\hline Headaches & 2 & 7 & 3 & 7 & 3 & 7 \\
\hline Laboratory investigations & 31 & 61 & 23 & 56 & 15 & 17 \\
\hline Injury and procedural & - & - & - & - & 5 & 5 \\
\hline Vascular disorders & - & - & - & - & 5 & 8 \\
\hline Psychiatric disorders & - & - & - & - & 7 & 7 \\
\hline Eye disorders & - & - & - & - & 3 & 5 \\
\hline Respiratory, thoracic and mediastinal disorders & - & - & - & - & 7 & 9 \\
\hline \multirow[t]{2}{*}{ General disorders and administrative-site conditions } & 0 & $7^{f}$ & 0 & $12^{f}$ & 9 & 7 \\
\hline & \multicolumn{2}{|c|}{ SAE's in $>1 \%$} & \multicolumn{2}{|c|}{ SAE's in $>1 \%$} & \multicolumn{2}{|c|}{ SAE's in $>1 \%$} \\
\hline Infections and infestations & 5.6 & 7.6 & 1.6 & 3.2 & 0.7 & 1.4 \\
\hline Spinal compression fracture & 5.5 & 1.9 & 1.6 & - & - & - \\
\hline Femoral neck fracture & - & - & 1.6 & - & - & - \\
\hline Malignancy & 0 & 1.9 & - & - & I.I & 0.3 \\
\hline
\end{tabular}

Notes: Values are the percent of patients rounded to nearest whole number for AE and the nearest one tenth for SAE. A blank represents no reported value. aDMARD

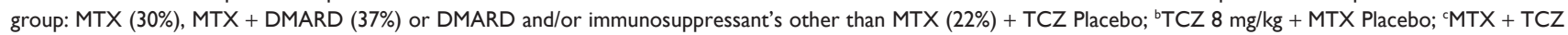
Placebo; MTX (7.5-20 mg escalating dose); 'TCZ $8 \mathrm{mg} / \mathrm{kg}$; ' Infusion reactions.

Abbreviations: AE, adverse event; SAE, serious adverse event; MSK, musculoskeletal; CT, connective tissue; SC, subcutaneous.

The primary endpoint was ACR20 response at week 24 (80.3\% for TCZ versus $52.5 \%$ for placebo, $P<0.001)$. Important secondary endpoints (Table 3 ) were the proportion of those with an ACR50/ACR70 response at week 24. Change from baseline in the DAS28, Modified Health Assessment Questionnaire (to represent significant clinical improvement) ${ }^{70}$ the proportion of those with clinical remission (DAS28 < 2.6), and good/moderate EULAR responses was assessed. Mean serum vascular endothelial growth factor was also checked in both groups.

SAMURAI ${ }^{54}$ was a 52 -week, Phase III, multicenter, randomized, x-ray reader-blinded, controlled Japanese evaluation of the ability of TCZ monotherapy to inhibit progression of structural joint damage in patients with early RA. Patients were randomly assigned to two groups. One groups received $\mathrm{TCZ} 8 \mathrm{mg} / \mathrm{kg}$ intravenously every four weeks $(\mathrm{n}=158)$ and the second group received conventional DMARD therapy $(\mathrm{n}=148)$; $30 \%$ were on MTX monotherapy, $37 \%$ were on a combination of MTX and DMARDs, and 22\% were on DMARDs and/or immunosuppressants other than MTX. A total of 306 patients were randomized, with 265 completing the study ( $85 \%$ in the TCZ group and $90 \%$ in the DMARD group).

The primary endpoint (Table 3 ) was changes in radiographic disease progression, and significantly less progression 
Table 6 Adverse events reported from the Phase III trials on tocilizumab combination therapy in rheumatoid arthritis

\begin{tabular}{|c|c|c|c|c|c|c|c|c|}
\hline & \multicolumn{3}{|c|}{ OPTION $^{57}$ (24 wks) } & \multicolumn{3}{|c|}{ RADIATE $^{58}$ (24 wks) } & \multicolumn{2}{|c|}{ TOWARD ${ }^{59}$ (24 wks) } \\
\hline & $\begin{array}{l}\text { MTX }^{a} \\
(n=204)\end{array}$ & $\begin{array}{l}\text { TCZ } 4^{b} \\
(n=2 \mid 2)\end{array}$ & $\begin{array}{l}\text { TCZ 8c } \\
(n=206)\end{array}$ & $\begin{array}{l}\text { MTX }^{a} \\
(n=158)\end{array}$ & $\begin{array}{l}\text { TCZ } 4^{\mathrm{b}} \\
(\mathrm{n}=16 \mathrm{I})\end{array}$ & $\begin{array}{l}\text { TCZ } 8^{c} \\
(n=170)\end{array}$ & $\begin{array}{l}\text { DMARD }^{d} \\
(n=4 I 4)\end{array}$ & $\begin{array}{l}\text { TCZ } 8^{e} \\
(n=802)\end{array}$ \\
\hline Any adverse event & 63 & 71 & 69 & 81 & 87 & 84 & 61 & 73 \\
\hline Serious adverse event & - & - & - & II & 7 & 6 & $4^{f}$ & $7^{f}$ \\
\hline Related serious adverse event & - & - & - & 2 & 2 & 3 & 1 & 3 \\
\hline AE leading to discontinuation & - & - & - & 5 & 6 & 6 & 2 & 4 \\
\hline$A E$ leading to dose modification & - & - & - & 8 & 15 & 7 & 7 & 14 \\
\hline \multirow[t]{2}{*}{ Death } & - & - & - & 0 & 0 & 0 & $<1$ & $<1$ \\
\hline & \multicolumn{3}{|c|}{ AE's in $\geq 5 \%$} & \multicolumn{3}{|c|}{ AE's in $>5 \%$} & \multicolumn{2}{|c|}{ AE's in $\geq 5 \%$} \\
\hline Infections and infestations & 27 & 31 & 32 & 41 & 47 & 49 & 32 & 37 \\
\hline Gastrointestinal disorders & 22 & 22 & 23 & 19 & 33 & 37 & 15 & 21 \\
\hline MSK + CT disorders & 16 & 14 & 12 & 21 & 21 & 15 & 18 & 13 \\
\hline Skin and SC tissue disorders & 7 & 13 & 18 & 14 & 31 & 22 & 7 & 17 \\
\hline Nervous system disorders & 13 & 12 & 15 & 17 & 20 & 18 & 9 & 12 \\
\hline Laboratory investigations & 5 & 12 & 15 & - & - & - & 3 & 12 \\
\hline Injury and procedural & - & - & - & 10 & 7 & 11 & 7 & 8 \\
\hline Vascular disorders & 5 & 8 & 6 & 5 & 11 & 8 & 5 & 7 \\
\hline Psychiatric disorders & - & - & - & 4 & 10 & 7 & - & - \\
\hline Eye disorders & - & - & - & 2 & 7 & 6 & - & - \\
\hline Metabolism and nutrition & - & - & - & 4 & 4 & 5 & - & - \\
\hline Hematologic & - & - & - & 3 & 3 & 5 & - & - \\
\hline $\begin{array}{l}\text { Respiratory, thoracic and } \\
\text { mediastinal disorders }\end{array}$ & 5 & 7 & 8 & 13 & 15 & 12 & 5 & 9 \\
\hline General disorders and & 7 & 9 & 11 & 14 & 16 & 12 & 7 & 8 \\
\hline & \multicolumn{3}{|c|}{ SAE's in $>1 \%$} & \multicolumn{3}{|c|}{ SAE's in $>1 \%$} & \multicolumn{2}{|c|}{ SAE's in > $1 \%$} \\
\hline Infections and infestations & 1.0 & 1.4 & 2.9 & 3.1 & 1.8 & 4.6 & 1.9 & 2.7 \\
\hline Gastrointestinal disorders & - & - & - & 1.3 & 1.2 & I.I & 0.2 & I.I \\
\hline MSK + CTD disorders & - & - & - & 3.1 & 1.2 & 0.6 & - & - \\
\hline
\end{tabular}

Notes: Values are the percent of patients rounded to nearest whole number for AE and the nearest one tenth for SAE. A blank represents no reported value. aMTX 10-25 mg + TCZ placebo; 'TCZ 4 mg/kg + MTX 10-25 mg; 'TCZ 8 mg/kg + MTX I0-25 mg; 'Permitted DMARD + TCX placebo ePermitted DMARD + TCX 8 mg/kg; $V$ alues represent $\%$ of SAE occurring in $\geq 3$ patients.

Abbreviations: AE, adverse event; SAE, serious adverse event; MSK, musculoskeletal; CT, connective tissue; SC, subcutaneous.

was seen in the TCZ group compared with the placebo group. Radiographic endpoints on modified Total Sharp Score ${ }^{71}$ erosion score, and joint space narrowing were assessed with a rank-transformed analysis of covariance on the change scores that included factors for baseline score and disease duration. ACR20, 50, and 70 responses, DAS28, and Modified Health Assessment Questionnaire scores were assessed using the intent-to-treat analysis.

\section{Combination therapy with tocilizumab and DMARDs}

OPTION $^{57}$ was a 24-week, Phase III, international (17 countries), randomized, double-blind, placebo-controlled, parallel group study of the efficacy and safety of TCZ in patients with active RA receiving background MTX therapy. Patients were randomly assigned to one of three groups. Two groups received TCZ intravenously every four weeks, ie, $4 \mathrm{mg} / \mathrm{kg}(\mathrm{n}=214)$ or $8 \mathrm{mg} / \mathrm{kg}(\mathrm{n}=205)$ plus MTX orally weekly (stable dose 10-25 mg). The third group received TCZ placebo intravenously every four weeks plus MTX orally weekly (stable dose 10-25 mg, $n=204$ ). Patients could be enrolled on rescue therapy of TCZ $8 \mathrm{mg} / \mathrm{kg}$ at week 16 . A total of 623 patients were randomized, with 566 completing the study, including 111 on rescue therapy. Ninety-three percent, $87 \%$, and $93 \%$ in the TCZ $8 \mathrm{mg} / \mathrm{kg}$, TCZ $4 \mathrm{mg} / \mathrm{kg}$ and placebo groups, respectively, completed the study.

The primary endpoint was ACR20 response at week 24, which was $59 \%$ for TCZ $8 \mathrm{mg} / \mathrm{kg}$ versus $26 \%$ for placebo $(P<0.001)$ and $48 \%$ for TCZ $4 \mathrm{mg} / \mathrm{kg}$ versus $26 \%$ for placebo $(P<0.001)$. Important secondary endpoints (Table 4$)$ were the proportion of patients with an ACR50/ACR70 response at week 24, change from baseline in DAS28, the proportion of those with a clinical remission (DAS28 $<2.6$ ), and a good/moderate EULAR response at week 24. Pain and patient's global assessment of disease activity were measured by a visual analog scale. HAQ-DI was used to 
assess physical function. The Short-Form 36 Health Survey $(\mathrm{SF}-36)^{72}$ and Functional Assessment of Chronic Illness Therapy (FACIT)-Fatigue assessment ${ }^{73}$ was used to assess health-related quality of life.

TOWARD ${ }^{59}$ was a 24 -week, international (18 countries), randomized, double-blind, placebo-controlled study which investigated the efficacy and safety of TCZ combined with conventional DMARDs in patients with active RA. Patients were randomly assigned in a ratio of 2:1 to two groups. One group received TCZ $8 \mathrm{mg} / \mathrm{kg}$ intravenously every four weeks plus stable DMARD therapy $(\mathrm{n}=805)$. The second group received TCZ placebo intravenously every four weeks plus stable DMARD therapy $(n=415)$. Patients could be enrolled in rescue therapy (adjustment/change of DMARD and/or glucocorticoids) at week 16 . A total of 1220 of patients were randomized, with $91 \%$ in the $\mathrm{TCZ}$ group and $78 \%$ in the placebo group completing the study.

The primary endpoint was ACR20 response at week 24 (61\% for TCZ $8 \mathrm{mg} / \mathrm{kg}$ versus $25 \%$ for placebo, $P<0.0001$ ). Important secondary endpoints (Table 4) were the proportion of those with an ACR50/ACR70 response at week 24, change from baseline of the DAS28, the proportion of those with a clinical remission (DAS28 <2.6) and a good/moderate EULAR response at week 24. The HAQ-DI was used to assess physical function. The SF-36 and FACIT-Fatigue assessment was used to assess healthrelated quality of life.

RADIATE $^{58}$ was a 24-week, international (US and Western Europe), randomized, double-blind, placebocontrolled, parallel-group Phase III evaluation of the efficacy and safety of TCZ in patients with active RA refractory to TNF- $\alpha$ inhibitor therapy. Patients were randomly assigned to three groups. Two groups received TCZ intravenously every four weeks, ie, $4 \mathrm{mg} / \mathrm{kg}(\mathrm{n}=163)$ or $8 \mathrm{mg} / \mathrm{kg}(\mathrm{n}=175)$ plus MTX orally weekly (stable dose $10-25 \mathrm{mg}$ ). The third group received TCZ placebo intravenously every four weeks plus MTX orally weekly (stable dose 10-25 mg, $\mathrm{n}=160$ ). Patients could be allocated to rescue therapy (TCZ $8 \mathrm{mg} / \mathrm{kg}$ plus MTX) at week 16. A total of 498 patients were randomized, with 417 completing the study and 113 being switched to rescue therapy. Within the study groups, $75 \%, 66 \%$, and $40 \%$ in the TCZ $8 \mathrm{mg} / \mathrm{kg}$, TCZ $4 \mathrm{mg} / \mathrm{kg}$, and placebo groups, respectively, completed the study.

The primary endpoint was ACR20 response at week 24 (50\% for TCZ $8 \mathrm{mg} / \mathrm{kg}$ versus $10.1 \%$ for placebo $[P<0.001]$ and $30.4 \%$ for TCZ $4 \mathrm{mg} / \mathrm{kg}$ versus $10.1 \%$ for placebo $[P<0.001])$. Important secondary endpoints (Table 4) were the proportion of patients with an ACR50/ACR70 response at week 24, change from baseline of the DAS28, the proportion of those with clinical remission (DAS28 < 2.6) and a good/ moderate EULAR response at week 24. The HAQ-DI was used to assess physical function.

\section{Safety and tolerability}

Data on adverse events and serious adverse events from the six fully published trials ${ }^{54-59}$ are shown in Tables 5 and 6. The TCZ prescribing information ${ }^{52}$ includes combined safety data from five trials, ${ }^{56-59,64} \mathrm{ie}, \mathrm{TCZ} 4 \mathrm{mg} / \mathrm{kg}$ in combination with MTX ( $\mathrm{n}=774)$, TCZ $8 \mathrm{mg} / \mathrm{kg}$ monotherapy $(\mathrm{n}=288)$, and TCZ $8 \mathrm{mg} / \mathrm{kg}$ in combination with DMARDs $(\mathrm{n}=1582)$. All patients in the registration studies who received at least one dose of TCZ have been included in the exposure population $(n=4009)$. Treatment was given to 3577 of these patients for at least six months. The study population was of mean age 52 years, with $82 \%$ being female and $74 \%$ Caucasian, and having moderate to severe active RA. ${ }^{52}$

The most commonly reported adverse events (occurring in $\geq 5 \%$ of patients on TCZ monotherapy and combination therapy) in the controlled studies ${ }^{56-59,64}$ up to six months were upper respiratory tract infections, nasopharyngitis, headache, hypertension, and increased alanine transaminase. Increased transaminase values and serious infections were the most common reason for discontinuation of the medication (5\% for patients taking TCZ and 3\% for patients taking placebo). ${ }^{52,56-59,64}$ The medication was generally well tolerated at doses of $4 \mathrm{mg} / \mathrm{kg}$ and $8 \mathrm{mg} / \mathrm{kg}$ given every four weeks.

Infections occurred at a rate of 119 events per 100 patientyears in the TCZ monotherapy group, which was similar to the rate in the MTX monotherapy group. The TCZ-DMARD combination group (4 mg/kg with 133 events and $8 \mathrm{mg} / \mathrm{kg}$ with 127 events) had events per 100 patient-years similar to those of the placebo-DMARD group (112 events). Upper respiratory tract infection and nasopharyngitis were the most commonly reported infections. The overall rate of infections with TCZ in the all-exposure population was 108 events per 100 patient-years. ${ }^{52,56-59,64}$

The most common serious infections were pneumonia, urinary tract infection, cellulitis, herpes zoster, gastroenteritis, diverticulitis, sepsis, and bacterial arthritis. The rate of serious infections in the TCZ monotherapy group was 3.6 per 100 patient-years compared with 1.5 per 100 patient-years in the MTX group. The TCZ-DMARD combination group ( $4 \mathrm{mg} / \mathrm{kg}$ with 4.4 events and $8 \mathrm{mg} / \mathrm{kg}$ with 5.3 events) had events per 100 patient-years similar to that in the placebo-DMARD 
group (3.9 events). The all-exposure population had an overall rate of serious infections of 4.7 events per 100 patient-years. The overall rate of fatal serious infections was low (0.13 per 100 patient-years). ${ }^{52,56-59,64}$

Infusion-related events were reported in $8 \%$ of patients on the $4 \mathrm{mg} / \mathrm{kg}$ TCZ-DMARD) combination, $7 \%$ on the $8 \mathrm{mg} / \mathrm{kg}$ TCZ-DMARD combination, and $5 \%$ on the placeboDMARD combination. Headache and skin reactions were the most commonly reported events within the first 24 hours of infusion. Hypertension was reported by $1 \%$ for both doses of TCZ during the infusion. A clinically significant hypersensitivity reaction was documented in $0.2 \%$ of patients $(9 / 4009)$ in the all-exposure population and this was usually observed during the second, third and/or fourth infusions. Anti-TCZ antibodies developed in $2 \%$ of patients (46/2876), and five had to withdraw from their allocated treatment because of a hypersensitivity reaction. ${ }^{52,56-59,64}$

Gastrointestinal perforations occurred at a rate of 0.26 events per 100 patient-years with TCZ therapy in the controlled clinical trials. ${ }^{52,56-59,64}$ Most of the patients who developed this complication were also taking nonsteroidal anti-inflammatory drugs, corticosteroids, and/or MTX, but whether this played a role in the gastrointestinal problems is not known. The allexposure population had an overall rate of 0.28 events per 100 patient-years for gastrointestinal perforation.

A decrease in neutrophil counts $\left(<1000 / \mathrm{mm}^{3}\right)$ occurred in $1.8 \%$ (TCZ $4 \mathrm{mg} / \mathrm{kg}$ ), and $3.4 \%$ (TCZ $8 \mathrm{mg} / \mathrm{kg}$ ) in the combination DMARD group..$^{52,56-59,64}$ The placebo group had only $0.1 \%$ of patients with low neutrophil counts. A decrease in counts below $500 / \mathrm{mm} 3$ occurred in $0.4 \%, 0.3 \%$, and $0.1 \%$ of patients in the $4 \mathrm{mg} / \mathrm{kg}$ and $8 \mathrm{mg} / \mathrm{kg} \mathrm{TCZ}$ and placebo groups (all with combination DMARDs), respectively. Platelets were also decreased $\left(<100,000 / \mathrm{mm}^{3}\right)$ as a result of TCZ-DMARD therapy in $1.3 \%(4 \mathrm{mg} / \mathrm{kg})$ and $1.7 \%(8 \mathrm{mg} / \mathrm{kg})$ of patients. Only $0.5 \%$ of patients in the placebo group developed a reduction in platelets, with no bleeding events in any of the groups. The all-exposure population had similar patterns as seen in the controlled trials. ${ }^{52,56-59,64}$

Reduction in the TCZ dose, interruption of TCZ treatment, or a reduced dose of DMARDs was needed in some patients because of liver enzyme abnormalities. There were more patients on a TCZ-DMARD combination than on TCZ monotherapy $(8 \mathrm{mg} / \mathrm{kg}$ ) or MTZ monotherapy who had elevations in aspartate transaminase and alanine transaminase. ${ }^{52}$

An elevation of lipids was seen at week 6, but these levels stabilized at this point and were responsive to lipidlowering agents. The mean LDL increased by $13 \mathrm{mg} / \mathrm{dL}$ (TCZ
$4 \mathrm{mg} / \mathrm{kg}$ + DMARD), $20 \mathrm{mg} / \mathrm{dL}$ (TCZ $8 \mathrm{mg} / \mathrm{kg}$ + DMARD), $25 \mathrm{mg} / \mathrm{dL}$ (TCZ $8 \mathrm{mg} / \mathrm{kg}$ monotherapy), and mean HDL increased by $3 \mathrm{mg} / \mathrm{dL}$ (TCZ $4 \mathrm{mg} / \mathrm{kg}$ + DMARD), $5 \mathrm{mg} / \mathrm{dL}$ (TCZ $8 \mathrm{mg} / \mathrm{kg}$ + DMARD), $4 \mathrm{mg} / \mathrm{dL}$ (TCZ $8 \mathrm{mg} / \mathrm{kg}$ monotherapy) in the controlled trials. ${ }^{52,56-59,64}$

Malignancy developed in 15 patients in the TCZ groups compared with eight patients in the control groups. The exposure-adjusted incidence was 1.32 events per 100 patientyears for the TCZ groups and 1.37 events per 100 patientyears for placebo-DMARD groups. A pooled analysis of long-term extension studies has not shown any new safety concerns in patients who have received this medication for an average of 2.4 years. $^{74}$

\section{Warnings and precautions}

TCZ should not be administered to those with active infection, those exposed to tuberculosis, or those with a history of serious or opportunistic infection. There is an increased risk of gastrointestinal perforation, and patients should seek medical attention for any new onset abdominal pain. Neutropenia, thrombocytopenia, elevated liver enzymes, and increased lipid parameters are all associated with TCZ use. Laboratory values should be monitored every $4-8$ weeks. Although the exact overall adverse impact of TCZ is not known, other warnings include risk of malignancy, hypersensitivity reactions, demyelinating disorders, hepatic impairment, and, of course, caution with live vaccinations..$^{52}$

\section{Regulatory progress}

TCZ has been approved in the US as monotherapy and in combination with MTX for the treatment of moderate to severely active RA in patients who have had an inadequate response to one or more TNF- $\alpha$ inhibitors. ${ }^{18}$ The recommended dose is $4 \mathrm{mg} / \mathrm{kg}$, with an increase to $8 \mathrm{mg} / \mathrm{kg}$ based on response, given every four weeks. ${ }^{52}$ The medication is also approved in Europe for the treatment of RA in those who have either responded inadequately to, or who were intolerant of, previous therapy with one or more DMARDs or a TNF- $\alpha$ inhibitor. ${ }^{19}$ TCZ is also approved in many other countries, including Japan, Mexico, India, Brazil, Switzerland, Australia, and the United Arab Emirates. ${ }^{75}$

\section{Conclusion}

This review has summarized the available evidence from randomized controlled trials of IL-6 inhibition in the treatment for RA. TCZ is effective in a number of patient groups with active RA, and has demonstrated significant 
clinical improvement in ACR response rates, individual ACR components, patient reported outcomes, radiographic progression, and DAS28 scores. Significant efficacy has been seen with both the $4 \mathrm{mg} / \mathrm{kg}$ and $8 \mathrm{mg} / \mathrm{kg}$ doses given every four weeks when compared with placebo.

TCZ monotherapy was superior to MTX monotherapy in those who had not had prior treatment failure and was significantly more effective then DMARDs in those who had prior treatment failure. Combination therapy with TCZ and MTX-DMARDs was significantly more effective than MTX or DMARDS as monotherapy. TCZ was also significantly effective in those who had previously failed a TNF- $\alpha$ inhibitor. Disease activity decreased and the quality of life improved in those who received TCZ, and there was sustainability of response shown in an extension trial. While showing efficacy as monotherapy and combination therapy with conventional DMARDs, the medication was generally well tolerated. Most of the adverse events were mild to moderate and comparable with placebo. The trials reviewed support the use of TCZ in active RA.

\section{Expert commentary}

The randomized controlled trials reviewed show the benefit of TCZ in active RA at the approved dose of 4-8 mg/kg every four weeks with or without combination MTX/DMARDs. The benefit of having a drug administered monthly as opposed to weekly or biweekly is an attractive option for patients and clinicians. Regarding the dose, when compared with placebo, TCZ patients were more likely to achieve higher ACR response rates with the $8 \mathrm{mg} / \mathrm{kg}$ dose than with the $4 \mathrm{mg} / \mathrm{kg}$ dose, and with similar adverse events.

TCZ is approved for use as monotherapy, as well as concomitantly with MTX/DMARDs in patients who have an inadequate response to a TNF- $\alpha$ inhibitor. DMARD combination therapy with biologics ${ }^{14,21,76}$ as well as biologic monotherapy ${ }^{77-79}$ has been shown to treat RA effectively in previous studies. It would be helpful to have a comparison of TCZ monotherapy with combination TCZ plus MTX. As monotherapy, this medication can be an option for those who cannot tolerate MTX unless the reason for intolerance is liver disease. If tolerability issues with MTX do not exist, most clinicians would consider continuing a patient on MTX when adding TCZ.

The radiographic progression was less in the TCZ group compared with placebo. However, this was only shown in one fully published monotherapy study. ${ }^{54}$ Data from the LITHE study has been shown at national conferences, and the fully published study is anticipated, and will reinforce the effect of TCZ on radiographic measures in RA.
The TCZ studies reviewed here used varying doses of MTX and this does limit comparison between the studies. Some of the patients were on low MTX doses that most clinicians would have titrated higher in a real world setting. This may have falsely placed some patients in a nonresponder group.

TCZ was effective in a fashion similar to the other approved biologic agents when compared with placebo. Bergman et $\mathrm{al}^{80}$ felt that the response was comparable with that of other biologic agents for ACR20 and ACR50, but greater for ACR70. However, it is difficult to compare TCZ with other biologics in previous studies because there are limitations to comparing separate studies with different populations and procedures. Comparison of different studies may hold more weight if a continuous response measure, such as DAS28, was used, but this was not available in all the compared trials. A head-to-head trial comparison of IL-6 inhibition and TNF- $\alpha$ inhibition would be interesting and help answer some questions regarding which therapies to resort to when conventional DMARDs fail.

The RADIATE ${ }^{58}$ study demonstrated TCZ efficacy after the failure of a TNF- $\alpha$ inhibitor, which is now the approved indication in the US and UK. It would be interesting to see how effective this medication is in those who have failed other biologics (abatacept and rituximab), as well as in patients stratified according to the DMARD they have failed. AMBI$\mathrm{TION}^{56}$ was the first to show biologic monotherapy superiority to MTX. There were a limited number of MTX-naïve patients in all these studies, and this will be an interesting group to look at in a future trial.

An advantage over some TNF inhibitors is that tuberculosis does not seem to be a safety issue because IL6 does not seem to have an effect on granuloma formation. All but one of the studies reviewed had the ACR20 as the primary outcome; this may have underestimated the difference between groups in terms of adverse events. Overall, the medication was safe to use as there were minimal adverse events and serious adverse events. However, future studies should be considerably longer, with a larger population, and include safety as a primary outcome. The study populations reported here did not have much comorbidity because these patients were excluded; safety may be an issue in sicker patients, and something to look into in future studies.

One safety issue that is concerning is the effect on LDL and total cholesterol. Coronary artery disease (CAD) is a leading cause of mortality and morbidity in the Western world, especially in the US, and a well-known extra-articular feature of RA is CAD. This topic may need to be investigated 
further in those with CAD, a family history of CAD, and/or hyperlipidemia. The current recommendation is to check lipid levels every six months, but this may need some modification in those with established CAD, diabetes mellitus, hyperlipidemia, and other comorbidities. Interestingly, IL-6 may be a biomarker of carotid artery atherosclerosis, ${ }^{81}$ and high levels of IL-6 have been a predictor of stroke in those with atrial fibrillation. ${ }^{82}$

TCZ has efficacy comparable with other biologics, and will be an option for those who fail DMARDs, TNF $\alpha$ inhibitors, and other biologic agents. There are many therapeutic options now, in contrast with a few years ago when clinicians only had a choice of three biologics. Before the widespread use of these newer biologic medications, clinicians should emphasize conventional DMARDs and short courses of glucocorticoids when appropriate. The exact time and which biologic to initiate is not always clear. Early aggressive therapy is recommended, so patients should be followed closely because it is not known on presentation who and what kind of patient will need treatment escalation. When escalation is needed, it could include combination DMARDs and/or biologic agents. The use of a second- and third-line biologic agent has become a standard after one or more has failed.

There are some guidelines put out by various agencies ${ }^{4-6,83}$ on RA management but there are still no standard algorithms on which biologic to use when there is DMARD failure or initial biologic failure. Experience shows that TNF- $\alpha$ inhibitors are used first-line, although there are many choices as the "next step" after failure of a TNF- $\alpha$ inhibitor. It will be difficult to know which biologic is superior for a patient with refractory disease without head-to-head trials comparing the different agents. At this point, it looks as though TCZ is a promising new agent for those who have failed biologics. Future studies will reveal the complete utility of this drug along with long-term safety issues. Until then, the use of this and other biologics is at the discretion of the provider based on each individual's disease characteristics.

\section{Five-year view}

TCZ monotherapy or combination therapy with DMARDs can achieve what other biologics have done, ie, improve clinical disease, inhibit radiographic progression, and improve quality of life for those with RA. There are many options in conventional DMARDs and/or biologics for clinicians in the treatment of active RA. Clinical trials comparing biologic agents may never be available to help clinicians decide which therapy is best for their patients. Most will continue to use what they have experience with and only use the newer agents for refractory disease unless one of those agents proves to be superior to their current practice tendencies.

Predicting which individual will respond to a particular DMARD and/or biologic will hopefully be the next forefront of therapy in RA. Biomarkers will help us identify which patients will respond to a given therapy from their initial presentation. We hope that one day rheumatologists will be able to provide optimal care for RA with a "first-line" agent on a case-by-case basis after looking at a biomarker profile.

\section{Disclosure}

The authors report no conflict of interests in this work.

\section{References}

1. Lee DM, Weinblatt ME. Rheumatoid arthritis. Lancet. 2001;358: 903-911.

2. Spector TD. Rheumatoid arthritis. Rheum Dis Clin North Am. 1990;16: 513-537.

3. Silman AJ, Pearson JE. Epidemiology and genetics of rheumatoid arthritis. Arthritis Res. 2002;4 Suppl 3:S265-S272.

4. National Institute for Health and Clinical Excellence. Rheumatoid Arthritis. National clinical guideline for management and treatment in adults. Available at: http://www.nice.org.uk/cg79. Accessed July 16, 2010.

5. Saag KG, Teng GG, Patkar NM, et al. American College of Rheumatology 2008 recommendations for the use of nonbiologic and biologic disease-modifying antirheumatic drugs in rheumatoid arthritis. Arthritis Rheum. 2008;59:762-784.

6. Emery P, van Vollenhoven R, Ostergaard M, et al. Guidelines for initiation of anti-tumour necrosis factor therapy in rheumatoid arthritis: Similarities and differences across Europe. Ann Rheum Dis. 2009;68: 456-459.

7. Tamura T, Udagawa N, Takahashi N, et al. Soluble interleukin-6 receptor triggers osteoclast formation by interleukin 6. Proc Natl Acad Sci USA. 1993;90:11924-11928.

8. Sack U, Kinne RW, Marx T, Heppt P, Bender S, Emmrich F. Interleukin-6 in synovial fluid is closely associated with chronic synovitis in rheumatoid arthritis. Rheumatol Int. 1993;13:45-51.

9. Gaujoux-Viala C, Smolen JS, Landewe R, et al. Current evidence for the management of rheumatoid arthritis with synthetic disease-modifying antirheumatic drugs: A systematic literature review informing the EULAR recommendations for the management of rheumatoid arthritis. Ann Rheum Dis. 2010;69:1004-1009.

10. Katchamart W, Trudeau J, Phumethum V, Bombardier C. Methotrexate monotherapy versus methotrexate combination therapy with nonbiologic disease modifying anti-rheumatic drugs for rheumatoid arthritis. Cochrane Database Syst Rev. 2010;4:CD008495.

11. Katchamart W, Trudeau J, Phumethum V, Bombardier C. Efficacy and toxicity of methotrexate (MTX) monotherapy versus MTX combination therapy with non-biological disease-modifying antirheumatic drugs in rheumatoid arthritis: A systematic review and meta-analysis. Ann Rheum Dis. 2009;68:1105-1112.

12. Svensson B, Boonen A, Albertsson K, van der Heijde D, Keller C, Hafstrom I. Low-dose prednisolone in addition to the initial diseasemodifying antirheumatic drug in patients with early active rheumatoid arthritis reduces joint destruction and increases the remission rate: A two-year randomized trial. Arthritis Rheum. 2005;52:3360-3370.

13. Kirwan JR. The effect of glucocorticoids on joint destruction in rheumatoid arthritis. The Arthritis and Rheumatism Council Low-Dose Glucocorticoid Study Group. N Engl J Med. 1995;333:142-146. 
14. Breedveld FC, Weisman MH, Kavanaugh AF, et al. The PREMIER study: A multicenter, randomized, double-blind clinical trial of combination therapy with adalimumab plus methotrexate versus methotrexate alone or adalimumab alone in patients with early, aggressive rheumatoid arthritis who had not had previous methotrexate treatment. Arthritis Rheum. 2006;54:26-37.

15. Emery P, Breedveld FC, Hall S, et al. Comparison of methotrexate monotherapy with a combination of methotrexate and etanercept in active, early, moderate to severe rheumatoid arthritis (COMET): A randomised, double-blind, parallel treatment trial. Lancet. 2008;372: 375-382.

16. Rantalaiho V, Korpela M, Laasonen L, et al. Early combination diseasemodifying antirheumatic drug therapy and tight disease control improve long-term radiologic outcome in patients with early rheumatoid arthritis: The 11-year results of the Finnish Rheumatoid Arthritis Combination Therapy trial. Arthritis Res Ther. 2010;12:R122.

17. Boers M. Cost-effectiveness of biologics as first-line treatment of rheumatoid arthritis: Case closed? Ann Intern Med. 2009;151:668-669.

18. US Food and Drug Administration. Website. Available at: http://www. fda.gov. Accessed July 21, 2010.

19. European Commission. Website. Available at: http://ec.europa.eu/ index_en.htm. Accessed July 21, 2010.

20. Maini R, St. Clair EW, Breedveld F, et al. Infliximab (chimeric anti-tumour necrosis factor alpha monoclonal antibody) versus placebo in rheumatoid arthritis patients receiving concomitant methotrexate: A randomised Phase III trial. ATTRACT Study Group. Lancet. 1999; 354:1932-1939.

21. van Vollenhoven RF, Ernestam S, Harju A, Bratt J, Klareskog L. Etanercept versus etanercept plus methotrexate: A registry-based study suggesting that the combination is clinically more efficacious. Arthritis Res Ther. 2003;5:R347-R351.

22. Weinblatt ME, Keystone EC, Furst DE, et al. Adalimumab, a fully human anti-tumor necrosis factor alpha monoclonal antibody, for the treatment of rheumatoid arthritis in patients taking concomitant methotrexate: The ARMADA trial. Arthritis Rheum. 2003;48:35-45.

23. Genovese MC, Bathon JM, Martin RW, et al. Etanercept versus methotrexate in patients with early rheumatoid arthritis: Two-year radiographic and clinical outcomes. Arthritis Rheum. 2002;46: $1443-1450$.

24. Lipsky PE, van der Heijde DM, St Clair EW, et al. Infliximab and methotrexate in the treatment of rheumatoid arthritis. Anti-Tumor Necrosis Factor Trial in Rheumatoid Arthritis with Concomitant Therapy Study Group. N Engl J Med. 2000;343:1594-1602.

25. St. Clair EW, van der Heijde DM, Smolen JS, et al. Combination of infliximab and methotrexate therapy for early rheumatoid arthritis: A randomized, controlled trial. Arthritis Rheum. 2004;50: 3432-3443.

26. Keystone EC, Kavanaugh AF, Sharp JT, et al. Radiographic, clinical, and functional outcomes of treatment with adalimumab (a human anti-tumor necrosis factor monoclonal antibody) in patients with active rheumatoid arthritis receiving concomitant methotrexate therapy: A randomized, placebo-controlled, 52-week trial. Arthritis Rheum. 2004;50:1400-1411.

27. Strehblow C, Haberhauer G, Fasching P. Comparison of different biologic agents in patients with rheumatoid arthritis after failure of the first biologic therapy. Wien Med Wochenschr. 2010;160:225-229.

28. van Vollenhoven R, Harju A, Brannemark S, Klareskog L. Treatment with infliximab (Remicade) when etanercept (Enbrel) has failed or vice versa: Data from the STURE registry showing that switching tumour necrosis factor alpha blockers can make sense. Ann Rheum Dis. 2003;62:1195-1198.

29. Hyrich KL, Lunt M, Watson KD, Symmons DP, Silman AJ. Outcomes after switching from one anti-tumor necrosis factor alpha agent to a second anti-tumor necrosis factor alpha agent in patients with rheumatoid arthritis: Results from a large UK national cohort study. Arthritis Rheum. 2007;56:13-20.
30. Keystone E, Heijde D, Mason D Jr, et al. Certolizumab pegol plus methotrexate is significantly more effective than placebo plus methotrexate in active rheumatoid arthritis: Findings of a fifty-two-week, Phase III, multicenter, randomized, double-blind, placebo-controlled, parallel-group study. Arthritis Rheum. 2008;58:3319-3329.

31. Fleischmann R, Vencovsky J, van Vollenhoven RF, et al. Efficacy and safety of certolizumab pegol monotherapy every 4 weeks in patients with rheumatoid arthritis failing previous disease-modifying antirheumatic therapy: The FAST4 WARD study. Ann Rheum Dis. 2009;68:805-811.

32. Smolen J, Landewe RB, Mease P, et al. Efficacy and safety of certolizumab pegol plus methotrexate in active rheumatoid arthritis: The RAPID 2 study. A randomised controlled trial. Ann Rheum Dis. 2009;68:797-804.

33. Smolen JS, Kay J, Doyle MK, et al. Golimumab in patients with active rheumatoid arthritis after treatment with tumour necrosis factor alpha inhibitors (GO-AFTER study): A multicentre, randomised, double-blind, placebo-controlled, phase III trial. Lancet. 2009;374:210-221.

34. Emery P, Kosinski M, Li T, et al. Treatment of rheumatoid arthritis patients with abatacept and methotrexate significantly improved healthrelated quality of life. J Rheumatol. 2006;33:681-689.

35. Kremer JM, Genant HK, Moreland LW, et al. Effects of abatacept in patients with methotrexate-resistant active rheumatoid arthritis: A randomized trial. Ann Intern Med. 2006;144:865-876.

36. Genovese MC, Becker JC, Schiff M, et al. Abatacept for rheumatoid arthritis refractory to tumor necrosis factor alpha inhibition. $N$ Engl $J$ Med. 2005;353:1114-1123.

37. Cohen SB, Emery P, Greenwald MW, et al. Rituximab for rheumatoid arthritis refractory to anti-tumor necrosis factor therapy: Results of a multicenter, randomized, double-blind, placebo-controlled, phase III trial evaluating primary efficacy and safety at twenty-four weeks. Arthritis Rheum. 2006;54:2793-2806.

38. Fleischmann RM, Schechtman J, Bennett R, et al. Anakinra, a recombinant human interleukin-1 receptor antagonist (r-metHuIL-1 ra), in patients with rheumatoid arthritis: A large, international, multicenter, placebo-controlled trial. Arthritis Rheum. 2003;48:927-934.

39. Thaler K, Chandiramani DV, Hansen RA, Gartlehner G. Efficacy and safety of anakinra for the treatment of rheumatoid arthritis: An update of the Oregon Drug Effectiveness Review Project. Biologics. 2009;3: 485-498.

40. Senolt L, Vencovsky J, Pavelka K, Ospelt C, Gay S. Prospective new biological therapies for rheumatoid arthritis. Autoimmun Rev. 2009;9: 102-107.

41. Church LD, McDermott MF. Canakinumab, a fully-human mAb against IL-1beta for the potential treatment of inflammatory disorders. Curr Opin Mol Ther. 2009;11:81-89.

42. Baslund B, Tvede N, Danneskiold-Samsoe B, et al. Targeting interleukin-15 in patients with rheumatoid arthritis: A proof-of-concept study. Arthritis Rheum. 2005;52:2686-2692.

43. Plater-Zyberk C, Joosten LA, Helsen MM, Koenders MI, Baeuerle PA, van den Berg WB. Combined blockade of granulocyte-macrophage colony stimulating factor and interleukin 17 pathways potently suppresses chronic destructive arthritis in a tumour necrosis factor alpha-independent mouse model. Ann Rheum Dis. 2009;68:721-728.

44. Leonardi CL, Kimball AB, Papp KA, et al. Efficacy and safety of ustekinumab, a human interleukin-12/23 monoclonal antibody, in patients with psoriasis: 76-week results from a randomised, doubleblind, placebo-controlled trial (PHOENIX 1). Lancet. 2008;371: 1665-1674.

45. Yago T, Nanke Y, Kawamoto M, et al. IL-23 induces human osteoclastogenesis via IL-17 in vitro, and anti-IL-23 antibody attenuates collagen-induced arthritis in rats. Arthritis Res Ther. 2007;9:R96.

46. Tak PP, Thurlings RM, Rossier C, et al. Atacicept in patients with rheumatoid arthritis: Results of a multicenter, Phase Ib, double-blind, placebo-controlled, dose-escalating, single- and repeated-dose study. Arthritis Rheum. 2008;58:61-72. 
47. Genovese MC, Kaine JL, Lowenstein MB, et al. Ocrelizumab, a humanized anti-CD20 monoclonal antibody, in the treatment of patients with rheumatoid arthritis: A phase I/II randomized, blinded, placebo-controlled, dose-ranging study. Arthritis Rheum. 2008;58: 2652-2661.

48. Robak T. Ofatumumab, a human monoclonal antibody for lymphoid malignancies and autoimmune disorders. Curr Opin Mol Ther. 2008;10: 294-309.

49. Kremer JM, Bloom BJ, Breedveld FC, et al. The safety and efficacy of a JAK inhibitor in patients with active rheumatoid arthritis: Results of a double-blind, placebo-controlled phase IIa trial of three dosage levels of CP-690,550 versus placebo. Arthritis Rheum. 2009;60: 1895-1905.

50. Weinblatt ME, Kavanaugh A, Burgos-Vargas R, et al. Treatment of rheumatoid arthritis with a Syk kinase inhibitor: A twelve-week, randomized, placebo-controlled trial. Arthritis Rheum. 2008;58: 3309-3318.

51. Cohen SB, Dore RK, Lane NE, et al. Denosumab treatment effects on structural damage, bone mineral density, and bone turnover in rheumatoid arthritis: A twelve-month, multicenter, randomized, doubleblind, placebo-controlled, phase II clinical trial. Arthritis Rheum. 2008;58:1299-1309.

52. Genentech Actemra ${ }^{\circledR}$ Prescribing Information. Available at: http://www. gene.com/gene/products/information/actemra/pdf/pi.pdf. Accessed July 1, 2010.

53. Hirano T, Taga T, Matsuda T, et al. Interleukin 6 and its receptor in the immune response and hematopoiesis. Int J Cell Cloning. 1990; 8 Suppl 1:155-166.

54. Nishimoto N, Hashimoto J, Miyasaka N, et al. Study of active controlled monotherapy used for rheumatoid arthritis, an IL-6 inhibitor (SAMURAI): Evidence of clinical and radiographic benefit from an $\mathrm{x}$ ray reader-blinded randomised controlled trial of tocilizumab. Ann Rheum Dis. 2007;66:1162-1167.

55. Nishimoto N, Miyasaka N, Yamamoto K, et al. Study of active controlled tocilizumab monotherapy for rheumatoid arthritis patients with an inadequate response to methotrexate (SATORI): Significant reduction in disease activity and serum vascular endothelial growth factor by IL-6 receptor inhibition therapy. Mod Rheumatol. 2009;19: $12-19$.

56. Jones G, Sebba A, Gu J, et al. Comparison of tocilizumab monotherapy versus methotrexate monotherapy in patients with moderate to severe rheumatoid arthritis: The AMBITION study. Ann Rheum Dis. 2010;69:88-96.

57. Smolen JS, Beaulieu A, Rubbert-Roth A, et al. Effect of interleukin-6 receptor inhibition with tocilizumab in patients with rheumatoid arthritis (OPTION study): A double-blind, placebo-controlled, randomised trial. Lancet. 2008;371:987-997.

58. Emery P, Keystone E, Tony HP, et al. IL-6 receptor inhibition with tocilizumab improves treatment outcomes in patients with rheumatoid arthritis refractory to anti-tumour necrosis factor biologicals: Results from a 24-week multicentre randomised placebo-controlled trial. Ann Rheum Dis. 2008;67:1516-1523.

59. Genovese MC, McKay JD, Nasonov EL, et al. Interleukin-6 receptor inhibition with tocilizumab reduces disease activity in rheumatoid arthritis with inadequate response to disease-modifying antirheumatic drugs: The tocilizumab in combination with traditional disease-modifying antirheumatic drug therapy study. Arthritis Rheum. 2008;58:2968-2980.

60. Choy EH, Isenberg DA, Garrood T, et al. Therapeutic benefit of blocking interleukin- 6 activity with an anti-interleukin- 6 receptor monoclonal antibody in rheumatoid arthritis: A randomized, doubleblind, placebo-controlled, dose-escalation trial. Arthritis Rheum. 2002; 46:3143-3150.

61. Nishimoto N, Yoshizaki K, Miyasaka N, et al. Treatment of rheumatoid arthritis with humanized anti-interleukin- 6 receptor antibody: A multicenter, double-blind, placebo-controlled trial. Arthritis Rheum. 2004; 50:1761-1769.
62. Maini RN, Taylor PC, Szechinski J, et al. Double-blind randomized controlled clinical trial of the interleukin-6 receptor antagonist, tocilizumab, in European patients with rheumatoid arthritis who had an incomplete response to methotrexate. Arthritis Rheum. 2006;54: 2817-2829.

63. Nishimoto N, Miyasaka N, Yamamoto K, Kawai S, Takeuchi T, Azuma J. Long-term safety and efficacy of tocilizumab, an antiIL-6 receptor monoclonal antibody, in monotherapy, in patients with rheumatoid arthritis (the STREAM study): Evidence of safety and efficacy in a 5-year extension study. Ann Rheum Dis. 2009;68: $1580-1584$.

64. Fleischmann R, Burgos-Vargas R, Ambs E, et al. Tocilizumab inhibits radiographic progression and improves physical function in rheumatoid arthritis patients at 2 years with increasing clinical efficacy over time. Proceedings of the American College of Rheumatology Annual Scientific Meeting. 2009 Oct 17-21; Philadelphia, PA.

65. Felson DT, Anderson JJ, Boers M, et al. American College of Rheumatology. Preliminary definition of improvement in rheumatoid arthritis. Arthritis Rheum. 1995;38:727-735.

66. Prevoo ML, van 't Hof MA, Kuper HH, van Leeuwen MA, van de Putte LB, van Riel PL. Modified disease activity scores that include twenty-eight-joint counts. Development and validation in a prospective longitudinal study of patients with rheumatoid arthritis. Arthritis Rheum. 1995;38:44-48.

67. Bruce B, Fries JF. The Stanford Health Assessment Questionnaire: A review of its history, issues, progress, and documentation. J Rheumatol. 2003;30:167-178.

68. Fransen J, van Riel PL. The Disease Activity Score and the EULAR response criteria. Clin Exp Rheumatol. 2005;23:S93-S99.

69. Farrar JT, Young JP Jr, LaMoreaux L, Werth JL, Poole RM. Clinical importance of changes in chronic pain intensity measured on an 11-point numerical pain rating scale. Pain. 2001;94:149-158.

70. Kosinski M, Zhao SZ, Dedhiya S, Osterhaus JT, Ware JE Jr. Determining minimally important changes in generic and disease-specific healthrelated quality of life questionnaires in clinical trials of rheumatoid arthritis. Arthritis Rheum. 2000;43:1478-1477.

71. van der Heijde DM, van Riel PL, Nuver-Zwart IH, Gribnau FW, vad de Putte LB. Effects of hydroxychloroquine and sulphasalazine on progression of joint damage in rheumatoid arthritis. Lancet. 1989;1: 1036-1038.

72. McHorney CA, Ware JE Jr, Raczek AE. The MOS 36-Item ShortForm Health Survey (SF-36): II. Psychometric and clinical tests of validity in measuring physical and mental health constructs. Med Care. 1993;31:247-263.

73. Cella D, Yount S, Sorensen M, Chartash E, Sengupta N, Grober J. Validation of the Functional Assessment of Chronic Illness Therapy Fatigue Scale relative to other instrumentation in patients with rheumatoid arthritis. J Rheumatol. 2005;32:811-819.

74. van Vollenhoven RF SD, Furie R, Krasnow I, Alecock E, Alten R. Longterm safety and tolerability of tocolizumab in patients with rheumatoid arthritis and a mean treatment duration of 2.4 years. Proceedings of the American College of Rheumatology Annual Scientific Meeting. 2009 Oct 17-21; Philadelphia, PA.

75. Roche Global Website ${ }^{\circledR}$. Available at: http://www.roche.com/investors/ ir_update/inv-update-2010-03-17.htm RGW. Accessed August 1, 2010.

76. Klareskog L, van der Heijde D, de Jager JP, et al. Therapeutic effect of the combination of etanercept and methotrexate compared with each treatment alone in patients with rheumatoid arthritis: Double-blind randomised controlled trial. Lancet. 2004;363:675-681.

77. Moreland LW, Schiff MH, Baumgartner SW, et al. Etanercept therapy in rheumatoid arthritis. A randomized, controlled trial. Ann Intern Med. 1999;130:478-486.

78. van de Putte LB, Atkins C, Malaise M, et al. Efficacy and safety of adalimumab as monotherapy in patients with rheumatoid arthritis for whom previous disease modifying antirheumatic drug treatment has failed. Ann Rheum Dis. 2004;63:508-516. 
79. Bathon JM, Martin RW, Fleischmann RM, et al. A comparison of etanercept and methotrexate in patients with early rheumatoid arthritis. $N$ Engl J Med. 2000;343:1586-1593.

80. Bergman GJ, Hochberg MC, Boers M, Wintfeld N, Kielhorn A, Jansen JP. Indirect comparison of tocilizumab and other biologic agents in patients with rheumatoid arthritis and inadequate response to disease-modifying antirheumatic drugs. Semin Arthritis Rheum. 2010;39:425-441.

81. Larsson PT, Hallerstam S, Rosfors S, Wallen NH. Circulating markers of inflammation are related to carotid artery atherosclerosis. Int Angiol. 2005;24:43-51.
82. Conway DS, Buggins P, Hughes E, Lip GY. Prognostic significance of raised plasma levels of interleukin-6 and C-reactive protein in atrial fibrillation. Am Heart J. 2004;148:462-466.

83. Smolen JS, Landewe R, Breedveld FC, et al. EULAR recommendations for the management of rheumatoid arthritis with synthetic and biological disease-modifying antirheumatic drugs. Ann Rheum Dis. 2010;69:964-975

\section{Publish your work in this journal}

Drug Design, Development and Therapy is an international, peerreviewed open-access journal that spans the spectrum of drug design and development through to clinical applications. Clinical outcomes, patient safety, and programs for the development and effective, safe, and sustained use of medicines are a feature of the journal, which has also been accepted for indexing on PubMed Central. The manuscript management system is completely online and includes a very quick and fair peer-review system, which is all easy to use. Visit http://www.dovepress.com/testimonials.php to read real quotes from published authors.

Submit your manuscript here: http://www.dovepress.com/drug-design-development-and-therapy-journal 